\title{
High-latitude plasma convection from Cluster EDI: variances and solar wind correlations
}

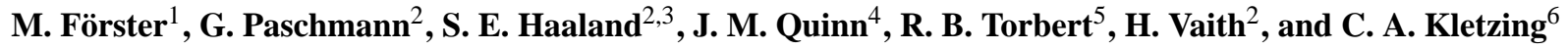 \\ ${ }^{1}$ GeoForschungsZentrum Potsdam, Potsdam, Germany \\ ${ }^{2}$ Max-Planck-Institut für extraterrestrische Physik, 85748 Garching, Germany \\ ${ }^{3}$ Department of Physics, University of Bergen, Norway \\ ${ }^{4}$ Boston University, Boston, MA 02215, USA \\ ${ }^{5}$ University of New Hampshire, Durham, NH 03824, USA \\ ${ }^{6}$ University of Iowa, Iowa City, IA 52242, USA
}

Received: 14 March 2007 - Revised: 21 June 2007 - Accepted: 16 July 2007 - Published: 30 July 2007

\begin{abstract}
Based on drift velocity measurements of the EDI instruments on Cluster during the years 2001-2006, we have constructed a database of high-latitude ionospheric convection velocities and associated solar wind and magnetospheric activity parameters. In an earlier paper (Haaland et al., 2007), we have described the method, consisting of an improved technique for calculating the propagation delay between the chosen solar wind monitor (ACE) and Earth's magnetosphere, filtering the data for periods of sufficiently stable IMF orientations, and mapping the EDI measurements from their high-altitude positions to ionospheric altitudes. The present paper extends this study, by looking at the spatial pattern of the variances of the convection velocities as a function of IMF orientation, and by performing sortings of the data according to the IMF magnitude in the GSM y-z plane, $\left|B_{y z}^{\mathrm{IMF}}\right|$, the estimated reconnection electric field, $E_{r, s w}$, the solar wind dynamic pressure, $P_{\mathrm{dyn}}$, the season, and indices characterizing the ring current $\left(D_{s t}\right)$ and tail activity (ASYM-H). The variability of the high-latitude convection shows characteristic spatial patterns, which are mirror symmetric between the Northern and Southern Hemispheres with respect to the IMF $B_{y}$ component. The latitude range of the highest variability zone varies with IMF $B_{z}$ similar to the auroral oval extent. The magnitude of convection standard deviations is of the same order as, or even larger than, the convection magnitude itself. Positive correlations of polar cap activity are found with $\left|B_{y z}^{\mathrm{IMF}}\right|$ and with $E_{r, s w}$, in particular. The strict linear increase for small magnitudes of $E_{r, s w}$ starts to deviate toward a flattened increase above about $2 \mathrm{mV} / \mathrm{m}$. There is also a weak positive correlation with $P_{\text {dyn }}$. At very small values of $P_{\mathrm{dyn}}$, a secondary maximum appears, which is even
\end{abstract}

Correspondence to: M. Förster

(mfo@gfz-potsdam.de) more pronounced for the correlation with solar wind proton density. Evidence for enhanced nightside convection during high nightside activity is presented.

Keywords. Ionosphere (Plasma convection) - Magnetospheric physics (Magnetospheric configuration and dynamics; Solar wind-magnetosphere interactions)

\section{Introduction}

Large spatial and temporal variability is a fundamental property of magnetospheric convection. This is mainly caused by variations in the driving solar wind and interplanetary magnetic field (IMF) conditions together with the complexity of the coupled system. Competing time-dependent processes are acting with various characteristic time scales such that steady-state conditions therefore rarely exist and any response to changes in various interplanetary conditions for any given moment in time depend further on the prior state of the system (Rostoker et al., 1988). The major part of the transfer of energy and momentum from the solar wind to Earth's magnetosphere and the basic high-latitude convection patterns are now known to be caused by reconnection between the IMF and Earth's geomagnetic field (Dungey, 1961) and to a minor extent by quasi-viscous interaction processes at the magnetopause (Axford and Hines, 1961). During periods when IMF has a southerly component, reconnection takes place on the dayside magnetopause. The newly reconnected field lines and the plasma attached to them are swept across the polar caps into the magnetotail where they eventually reconnect again. Both of these two basic timedependent components of the magnetospheric convection cycle can be reasonably parametrized in terms of the concurrent

Published by Copernicus Publications on behalf of the European Geosciences Union. 


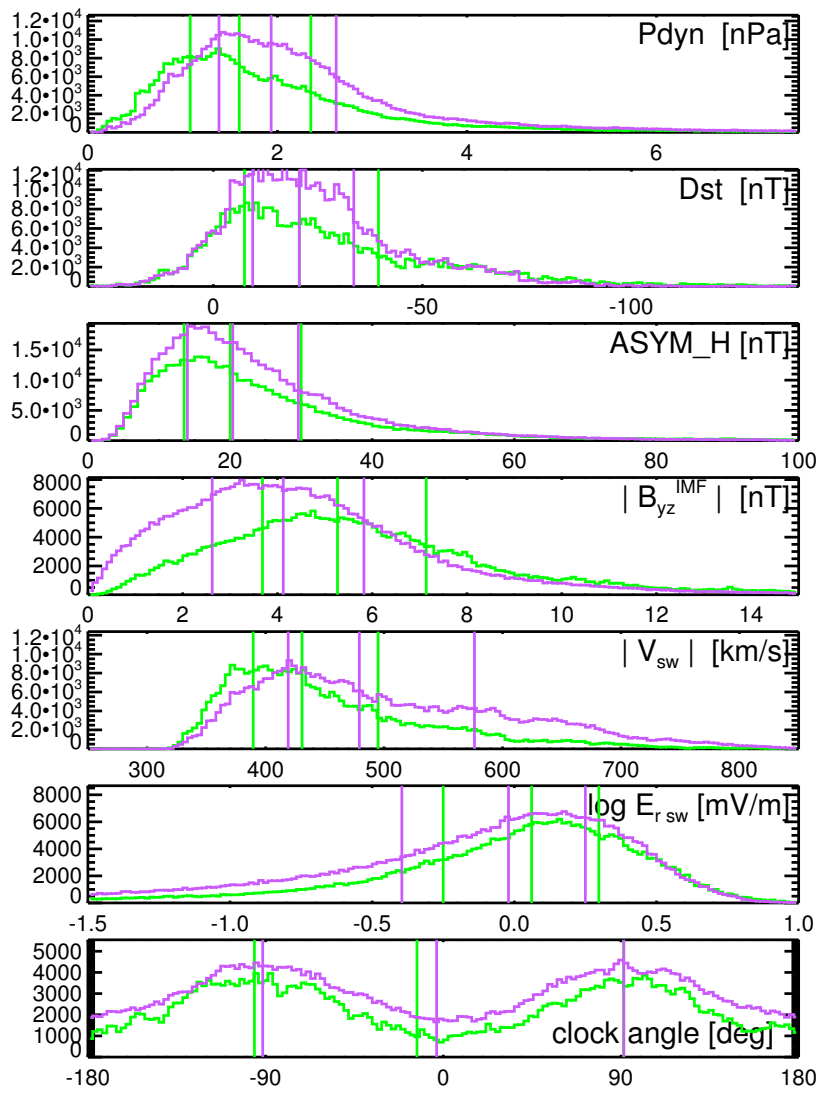

Fig. 1. Solar wind and geomagnetic parameter distribution for the time interval of EDI observations 2001-2006. All data points used for the analysis, i.e., after filtering for stable IMF conditions, are drawn as green lines; the purple lines illustrate the contrasting data set of all those data points that were removed by the filtering. The panels from top to bottom show the dynamic pressure of the solar wind protons, $P_{\text {dyn }}$, at the magnetopause, the $D_{s t}$ index characterizing geomagnetic activity of the ring current, the ASYM-H index which is used here as a proxy of the $A E$ index of auroral geomagnetic activity, the magnitude of the IMF vector components in the GSM y-z-plane, $\left|B_{y z}^{\mathrm{IMF}}\right|$, the magnitude of solar wind velocity, $\left|V_{s . w .}\right|$, the solar wind reconnection electric field, $E_{r, s w}$ (see Eq. 3), and finally the IMF clock angle. The vertical lines in each panel indicate the quartiles of the distribution for the corresponding parameter.

near-Earth interplanetary conditions, although with different response times (Cowley and Lockwood, 1992). For northward IMF, reconnection takes place at high latitudes between the IMF and Earth's lobe field (see, e.g., Burke et al., 1979; Reiff and Burch, 1985; Reiff and Heelis, 1994).

The result of these processes is a large scale circulation of plasma in Earth's magnetosphere. Since the geomagnetic field lines are nearly equipotentials, and the large scale circulation map to high magnetic latitudes, this plasma circulation and its variability is also manifested in the high latitude ionosphere. In addition, the ionosphere itself and the thermosphere where it is embedded play a role. Sunlight and particle precipitation enhances the ionospheric conductivity leading to an attenuation of the electric field, and the ionosphere, being tightly coupled to the thermosphere, acts as a "drag" on convection (Cole, 1963; Hill, 1976). The highlatitude convection and its variability in particular also play a major role in Joule heating of the ionosphere and thermosphere. Joule heating is generated due to relative motions of the neutral gas and ionized components, being proportional to the square of the velocity difference. The contribution of the irregular part of the convection has been shown to be comparable to or in certain regions even larger than the Joule heating of the averaged or background convection. It is as an essential contributor of thermospheric energy at high latitudes (Codrescu et al., 1995, 2000; Crowley and Hackert, 2001). The inertia of the thermospheric neutral winds can help to maintain the ionospheric convection independently of the magnetospheric driver processes and is known as the fly-wheel effect (Banks, 1972; Coroniti and Kennel, 1973). Taken together, the convection patterns are thus caused by the combined effect of several processes rather than a single elementary process (Tanaka, 2001). Statistical dependencies of the solar wind drivers such as the IMF and the solar wind dynamic pressure, have been studied extensively for several decades (e.g., Reiff et al., 1981; Heppner and Maynard, 1987; Boyle et al., 1997).

In an earlier paper (Haaland et al., 2007), henceforth referred to as Paper 1, the statistical pattern of high-latitude (magnetic latitudes $>58^{\circ}$ ) ionospheric convection as a function of the IMF clock angle, deduced from measurements by the Electron Drift Instruments (EDI) onboard the Cluster satellites during the years 2001-2006, was presented. Paper 1 describes the method, consisting of an improved technique for calculating the propagation delay between the solar wind monitor, the Advanced Composition Explorer (ACE), and Earth's magnetosphere, filtering the data for periods of sufficiently stable IMF orientations, and mapping the EDI measurements from their high-altitude positions to ionospheric altitudes. In Paper 1 we sorted the data according to the IMF orientation, averaged the data, and generated maps of the potential distribution. In the present paper we use the same comprehensive data set to investigate the dependencies of high-latitude convection on various other solar wind and magnetospheric activity parameters. We also discuss the spatial pattern of the variances of the convection velocities.

\section{Data set characteristics}

In order to characterize the data set, Fig. 1 shows the distributions of the selected solar wind parameters over the time-span of our data set: clock angle $\theta$, magnitude of the IMF in the GSM (y,z)-plane $\left|B_{y z}^{\mathrm{IMF}}\right|$ (sometimes called $B_{T}$ or "transverse IMF magnitude"), the solar wind speed $\left|V_{\text {s.w. }}\right|$, the magnitude of the solar wind electric field projected along the reconnection line at the dayside magnetopause, $E_{r, s w}$, and dynamic 
pressure $P_{\text {dyn }}$ of solar wind protons (including heavier ions like alpha particle gives on average about $20 \%$ higher values). The parameters were derived from measurements at ACE as described in Paper 1. Figure 1 also shows the distribution of two geomagnetic indices ( $D_{s t}$ and ASYM-H) assembled together with the solar wind and IMF parameters with the same 1-min resolution. The solar wind parameters have been shifted for arrival time at the magnetopause (assumed to be located at $\mathrm{X}_{\mathrm{GSE}}=10 R_{E}$ ).

The method described in Paper 1 used a bias vector filtering to select relatively stable IMF conditions for well-defined magnetospheric convection response patterns as a function of the IMF clock angle in eight discrete sectors with $45^{\circ}$ width each. IMF sector 0 is purely northward (IMF $B_{z}+$ ) directed, the other sectors follow clockwise in the IMF $y-z$ plane, so that IMF sectors 2 and 6 correspond to $B_{y}+$ and $B_{y}-$, respectively, and sector 4 is close to strict southward direction (IMF $B_{z}-$ ). This selection process resulted in a filtered data set which comprises nearly half the full data set originally available. However, the IMF stability filtering might have biased the characteristic solar wind conditions in a systematic manner. To check this, we plotted in Fig. 1 not only the distributions of the data set with stable IMF conditions used for our study (green lines), but also the complementary ones, i.e., the distributions for all rejected data points (purple lines). The vertical lines indicate the quartiles of the distribution.

Differences between the two data sets are clearly seen in the solar wind parameter $P_{\text {dyn }}$, the IMF magnitude $\left|B_{y z}^{\mathrm{IMF}}\right|$, and the solar wind velocity $\left|V_{\text {s.w. }}\right|$. The discarded data points are characterized by larger average solar wind speed and therefore larger mean $P_{\text {dyn }}$ values while the proton density distribution (not shown) is generally not influenced. The $D_{s t}$ index becomes more concentrated within the range $-10 \mathrm{nT}$ to $-35 \mathrm{nT}$ under disturbed IMF conditions while the partial ring current activity index ASYM-H as well as the average clock angle distribution of the IMF vectors do not show any significant difference.

The parameters we have chosen for our secondary sortings are not independent of the IMF clock angle that we used to sort the data in Paper 1, and they are also somewhat dependent on each other. This is illustrated by Table 1. For a significance test of the correlation coefficients we used the Student's t-distribution. Due to the large number of data points, all correlation coefficients estimated can be regarded as statistically reliable. Their significance in a pure statistical sense is already gained for values above $\approx 0.01$. The quality of correlation, on the other hand, is given by the correlation coefficient itself with its range from -1.0 to +1.0 ; larger magnitudes indicate stronger correlations. To guide the reader, we highlighted in Table 1 all coefficients with magnitudes $>0.5$.

The clear anticorrelation between $B_{z}^{\mathrm{IMF}}$ and the absolute value of the clock angle $|\theta|$ results simply from its definition as function of $B_{y}^{\mathrm{IMF}}$ and $B_{z}^{\mathrm{IMF}}$. A further clear anticorrelation

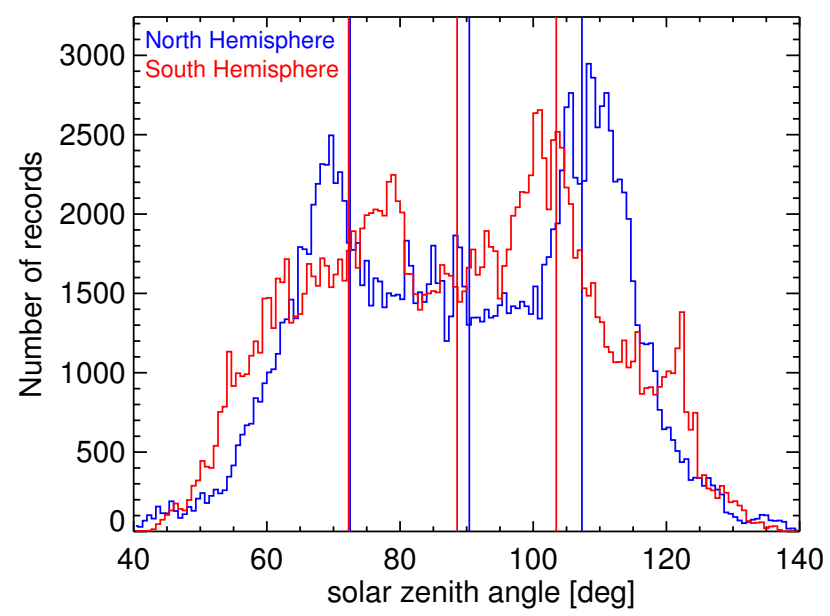

Fig. 2. Solar zenith angle distributions at the ionospheric footpoint location of the mapped EDI drift vectors in the ionosphere for North (blue) and South (red) Hemisphere separately. The vertical lines indicate the quartiles of the distributions.

exists between the geomagnetic indices $D_{s t}$ and ASYM-H; declining ring current values of disturbed periods are related to a rising auroral activity. All other high correlation values concern the reconnection electric field parameter of the solar wind $E_{r, s w}$, except for its relation to $P_{\text {dyn }}$ and $\left|V_{s w .}\right|$. There are also some smaller, but noticeable correlations of ASYM$\mathrm{H}$ with $B_{z}^{\mathrm{IMF}}$ and $\left|B_{y z}^{\mathrm{IMF}}\right|$. All other pairs of parameters are uncorrelated.

The line of apsides of the Cluster orbit, originally equatorial, is tilting further and further southward with time. In our data set, there is therefore an asymmetry in the NorthSouth coverage within the high magnetic latitudes regions. This manifests itself, for example, in different seasonal coverages at the Northern and Southern Hemisphere (see Fig. 5 in Paper 1, middle and bottom panels). Also, Southern Hemisphere data are on average obtained at higher altitudes $\left(9 R_{E}\right.$ versus $7 R_{E}$ for the Northern Hemisphere). However, the Southern Hemisphere data contain a larger fraction of observations taken during sunlit conditions. This is probably due to a clear difference in the offsets between the geographic and geomagnetic poles at the North and South Hemisphere. Based on the International Geomagnetic Reference Field (IGRF) model, the 2005 location of the north magnetic pole is $83.21^{\circ} \mathrm{N}$ and $118.32^{\circ} \mathrm{W}$ and the south magnetic pole is $64.53^{\circ} \mathrm{S}$ and $137.86^{\circ} \mathrm{E}$. The larger displacement at the Southern Hemisphere leads to a larger diurnal "wobbling" of the polar cap area with respect to the geographic coordinates. Figure 2 shows the number of observations for the Northern (blue lines) and Southern (red lines) Hemisphere versus solar zenith angle. Zenith angles below approximately $100^{\circ}$ corresponds to a sunlit polar cap ionosphere and thus enhanced ionization and subsequent attenuation of the electric field. As already pointed out by Cole (1963) and Hill (1976), a higher 
Table 1. Correlations between the various driver parameters (using all bias-filtered data).

\begin{tabular}{lrrrrrrrr}
\hline \multicolumn{8}{c}{ Driver parameter } \\
\hline & $\left|V_{s, w .}\right|$ & \multicolumn{1}{c}{$B_{z}^{\mathrm{IMF}}$} & $\left|B_{y z}^{\mathrm{IMF}}\right|$ & $E_{r, s w}$ & $P_{\text {dyn }}$ & $D_{s t}$ & ASYM-H & $|\theta|^{*}$ \\
\hline$\left|V_{s w . .}\right|$ & 1 & & & & & & & \\
$B_{z}^{\mathrm{IMF}}$ & -0.002 & 1 & & & & & & \\
$\left|B_{y z}^{\mathrm{IMF}}\right|$ & 0.148 & -0.045 & 1 & & & & & \\
$E_{r, s w}$ & 0.273 & $-\mathbf{0 . 7 2 7}$ & $\mathbf{0 . 6 5 7}$ & 1 & & & & \\
$P_{\text {dyn }}$ & 0.160 & 0.123 & 0.478 & 0.218 & 1 & & & \\
$D_{s t}$ & -0.289 & 0.360 & -0.322 & $-\mathbf{0 . 5 0 3}$ & -0.068 & 1 & & \\
ASYM-H & 0.290 & -0.443 & 0.468 & $\mathbf{0 . 6 6 2}$ & 0.242 & $-\mathbf{0 . 5 9 8}$ & 1 & \\
$|\theta|^{*}$ & -0.013 & $-\mathbf{0 . 7 9 2}$ & 0.035 & $\mathbf{0 . 5 3 4}$ & -0.069 & -0.312 & 0.349 & 1 \\
\hline${ }^{*}$ absolute values of the clock angle used. & & & & & &
\end{tabular}

ionospheric conductivity will retard the convection due to the drag force caused by ion-neutral collisions. Since the magnetic field lines are nearly equipotentials, the effect of this drag is also observed at Cluster altitudes. Due to the asymmetry, the average convection velocities obtained within the Southern polar cap are slightly lower $(\approx 7 \%)$ than those from the Northern Hemisphere. This difference is smaller within the very central part of the polar cap, where the mapped EDI drift data have best coverage.

\section{Convection variability}

In Paper 1, we have presented the average convection patterns as a function of IMF direction without any considerations of variability. But as noted earlier, the convection pattern is known to be highly variable on different timescales. Variations on timescales of minutes are known even for relatively stable IMF conditions (Bristow et al., 2004).

To characterize the variability of the convection in our statistical study of EDI drift measurements, we used two different variances. Normalized to the average drift magnitude, they are defined as follows:

$\sigma_{\text {total }}^{2}=\frac{\left\langle|\boldsymbol{v}|^{2}\right\rangle-|\langle\boldsymbol{v}\rangle|^{2}}{\left\langle|\boldsymbol{v}|^{2}\right\rangle}$
$\sigma_{\text {mag }}^{2}=\frac{\left\langle|\boldsymbol{v}|^{2}\right\rangle-\langle|\boldsymbol{v}|\rangle^{2}}{\left\langle|\boldsymbol{v}|^{2}\right\rangle}$

where $\langle\ldots\rangle$ denotes average over time and $v$ is the velocity vector. The first variance, $\sigma_{\text {total }}^{2}$, is the normalized variance (equivalent to the sum over the component variances) of the total velocity vector. It represents the variability of the full vector, with a steady pointing direction yielding zero variance. The second variance, $\sigma_{\mathrm{mag}}^{2}$, is the normalized variance of the velocity magnitude, and gives the average deviation of the velocity magnitude from its local average value. Both variances range from 0 to 1 .
The normalized total drift velocity variance, $\sigma_{\text {total }}^{2}$, is shown in Figs. 3 and 4 for the Northern and Southern Hemisphere, respectively. The eight panels are for different clock angle ranges (corresponding to those in Paper 1) and reveal a systematic pattern of the drift variance over the whole polar cap as a function of the IMF clock angle. The variability approaches 1 over large areas in a systematic way. For the three sectors of northward IMF (upper row), this enhanced variability fills nearly the whole polar cap region, while for southward IMF (bottom panels), the variance is small within the polar cap.

The $B_{y}^{\mathrm{IMF}}$ dominated sectors ( 2 and 6 ) show a dawn-dusk asymmetry in the maximum intensities of the normalized variance. The normalized variance at the Northern Hemisphere (Fig. 3) shows high values at the dusk (dawn) side for positive (negative) $B_{y}^{\mathrm{IMF}}$ values. The dayside quadrant at the dusk (dawn) side is completely covered while this enhanced normalized variance is confined to about 20:00 MLT (03:00 MLT) on the nightside. Note that regions inside the polar cap with normalized variances close to 1 have lower average convection velocities. They correspond to the roundshaped convection cells areas with wider spaced potential contours (see Figs. 7 and 8 in Paper 1). For northward IMF, somewhat broader regions of more enhanced variability are seen on the dayside as, e.g., in sector 1 of Fig. 3 and sector 7 of Fig. 4.

Inside the polar cap region, there is a region of more structured flow (smaller normalized variances and more 'bundled' convection stream lines) which broadens for further southward turning of the IMF as seen in the lower row (sectors 35). For purely southward IMF (Sector 4), the well-organized transpolar flow covers the entire polar cap region down to nearly $70^{\circ}$. Further equatorward, in the convection reversal zone and return flow, the variance is higher than in the central polar cap again. The diameter of the low-variance region in the central polar cap becomes smaller for northward turning IMF. 

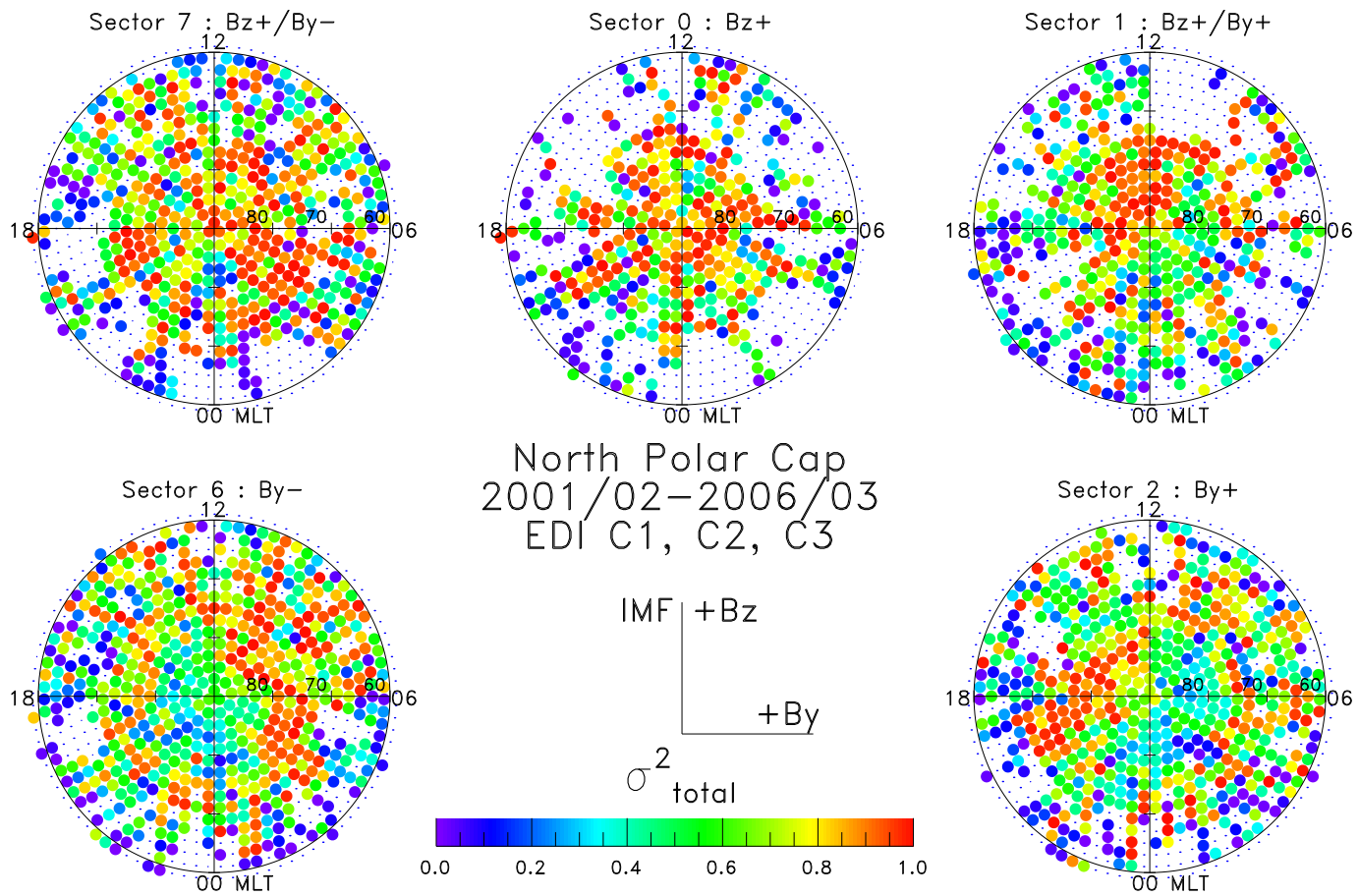

North Polar Cap
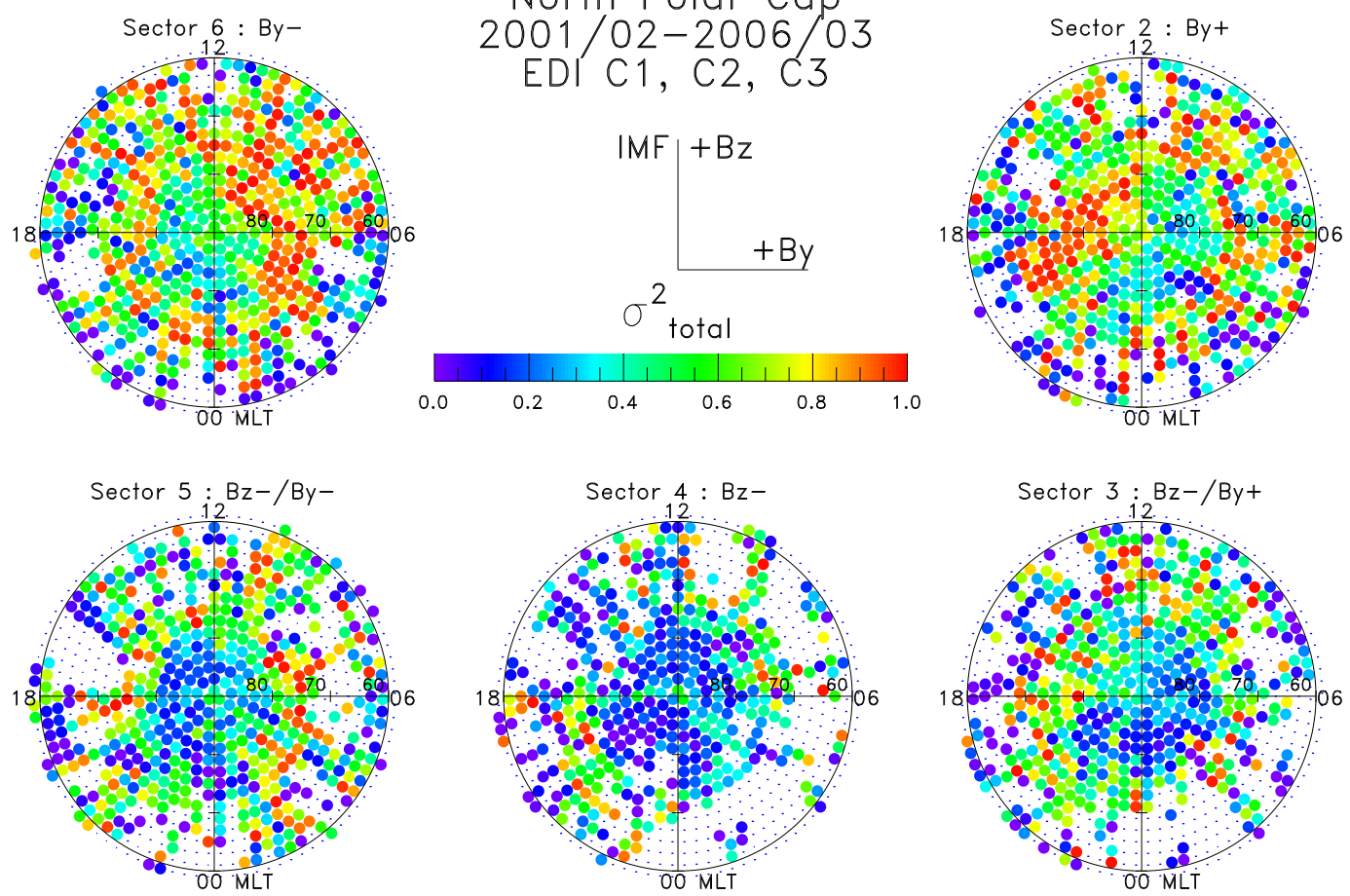

Fig. 3. Total normalized variance of the EDI drift vectors mapped to the Northern high latitude ionosphere for the time interval from February 2001 to March 2006. The outer circle of each panel represents $60^{\circ}$ magnetic latitude and the numbers indicate magnetic local time (MLT). The color bar shows the intensity of the variability; the normalized values are confined to an interval between 0 and 1 . The variance is calculated for each bin individually, based on at least three valid mapped drift vectors within the bin.

Comparing the Southern Hemisphere (Fig. 4) with the Northern (Fig. 3), the patterns are mirror symmetric with respect to the $B_{y}^{\mathrm{IMF}}$ dependence, that is, there are high values of normalized variance at the dawn (dusk) side for positive (negative) $B_{y}^{\mathrm{IMF}}$ values, respectively. All other differences are minor as, e.g., an apparently broader ring of enhanced variability at auroral and subauroral latitudes on the nightside for southward IMF.

The other normalized variance, $\sigma_{\text {magn }}^{2}$ (not shown here), reveals similar patterns of variability with the same $B_{y}^{\mathrm{IMF}}$ dependence, but on a much lower level of amplitudes. Its maximum magnitudes reach only about half of those of the total variance.
Figures 3 and 4 were for the normalized variances. To emphasize the absolute variations, Fig. 5 shows the standard deviation of the full vector for the Northern Hemisphere, without any normalization.

The auroral oval (convection reversal zone) and its diameter change with varying $B_{z}^{\mathrm{IMF}}$ values is clearly reflected in these patterns. For sector 0 (purely northward IMF), this oval has its smallest radial extent and the region of enhanced standard deviation values is nearly closed throughout the polar cap. This oval gradually extends for the other sectors with a maximum diameter low values (more ordered convection) within the polar cap for sectors 3-5 under southward IMF conditions. The absolute values within the auroral oval are of the order of the average drift velocity and are marked by the 

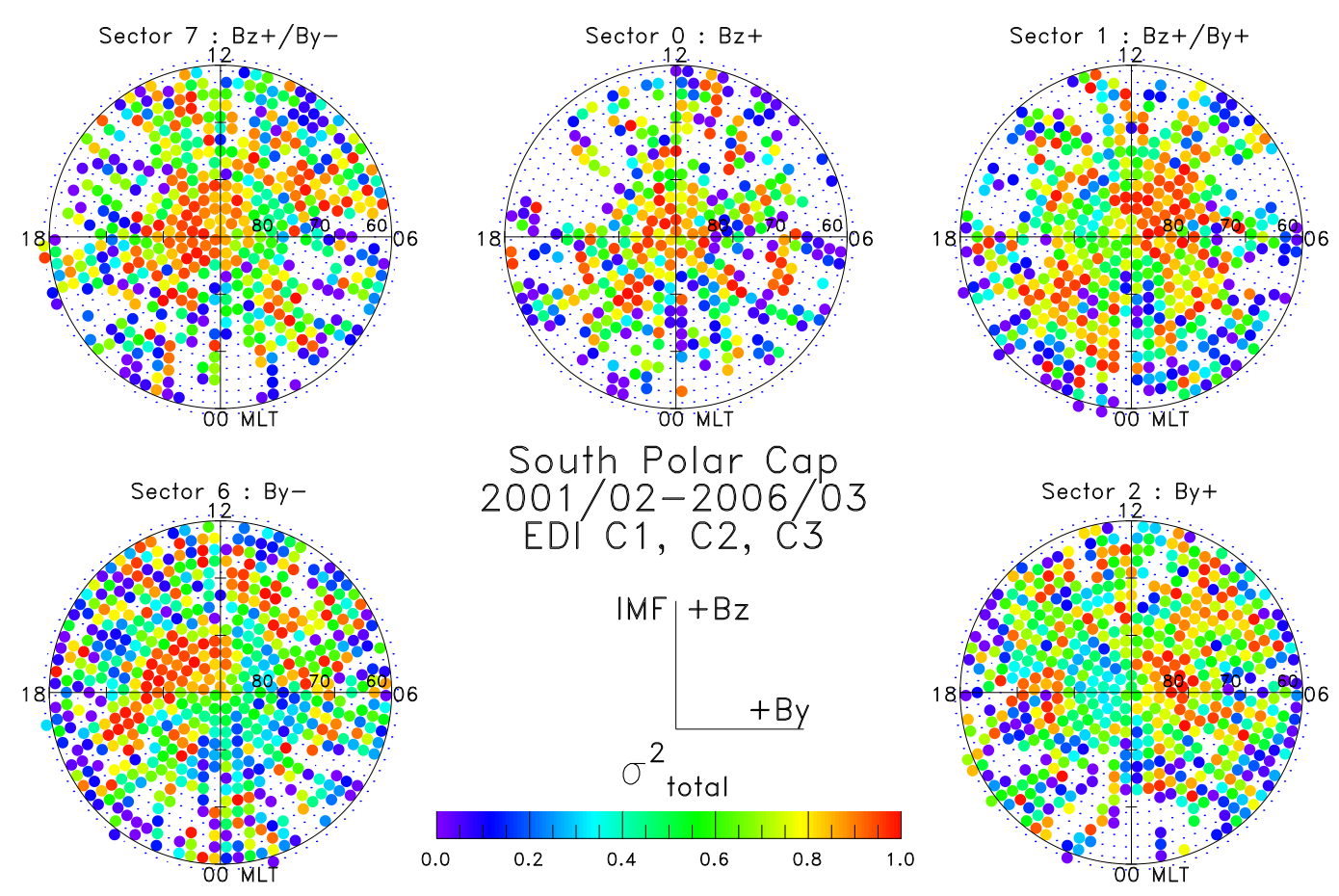

South Polar Cap
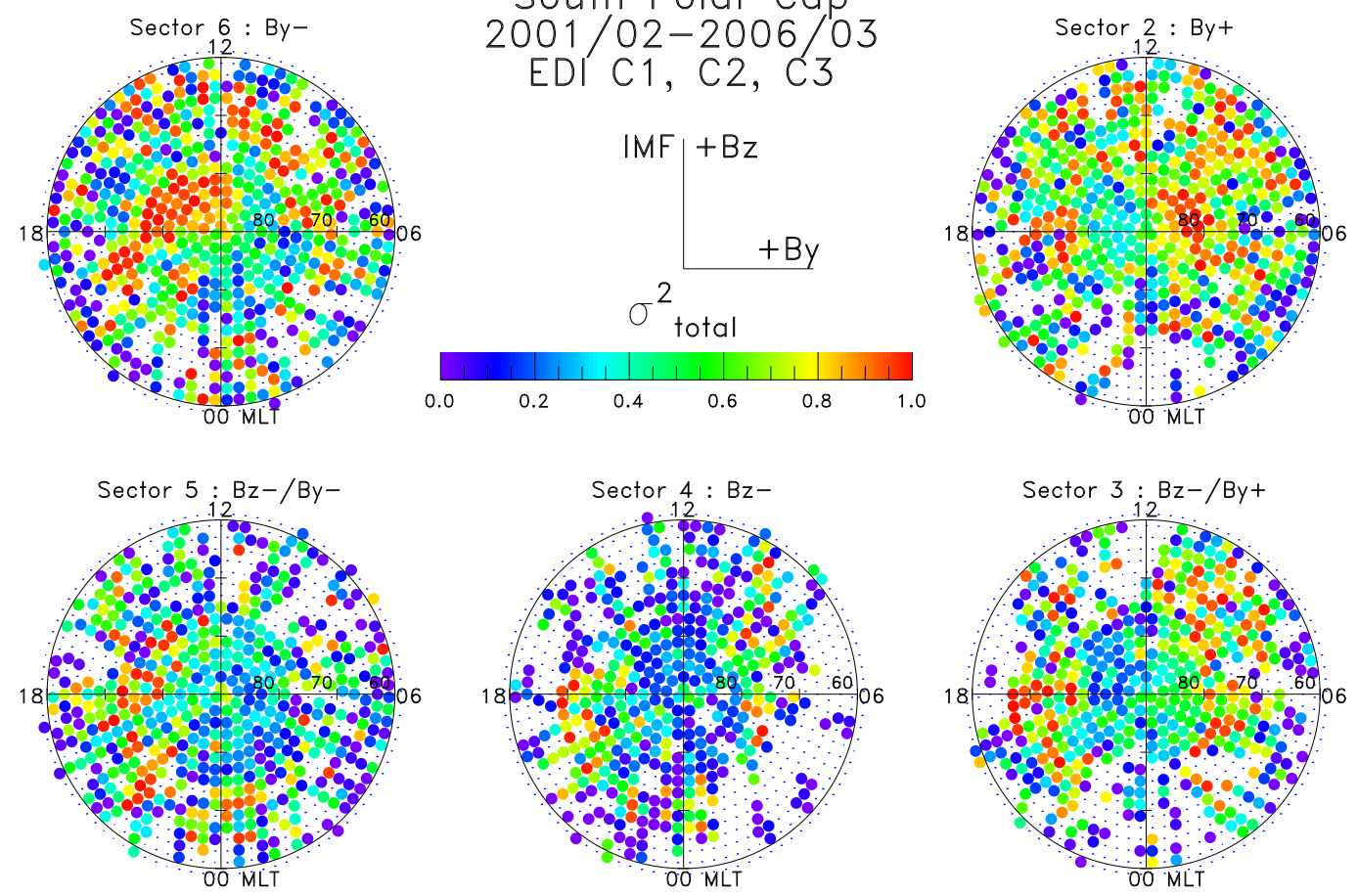

Fig. 4. As in Fig. 3, but for the Southern Hemisphere.

range of the colour scale chosen (about $700 \mathrm{~m} \mathrm{~s}^{-1}$ ). Within the auroral oval inside the central polar cap the standard deviations have about half of this magnitude (around $350 \mathrm{~m} \mathrm{~s}^{-1}$ ). The $B_{y}^{\mathrm{IMF}}$ dependence that was apparent in the normalized variances is not seen here in the standard deviation plot.

On the nightside, there is a tendency for enhanced values in the evening to midnight magnetic local times, in particular for southward IMF, extending toward early morning hours $(\approx 02: 00 \mathrm{MLT})$ as can be seen in sector 5 (this cannot be confirmed for sectors 3 and 4 due to scarcity of mapped EDI drift vectors in this area). This might be due to enhanced substorm activity.

While Figs. 3-5 characterize the variability in terms of normalized variances and unnormalized standard deviations, Fig. 6 shows time series of the $\mathrm{X}_{S M}$ component of all available EDI velocity data that are mapped into the central polar cap region poleward of $80^{\circ}$ magnetic latitude, divided into four different main IMF directions corresponding to the four 90-deg quadrants of IMF clock angle. Particular striking is that there is a large variability down to very short time scales, in particular for northward IMF (quadrant 0 - top panel), but also for IMF directions in the ecliptic plane (quadrants 1 and 3 ). For southward IMF (quadrant 2 ) the variability is much lower, and comparable to or even smaller than the average drift vector magnitude (see the red lines in Fig. 6). Table 2 lists additionally the mean values and standard deviations of the the boxcar averages shown in Fig. 6. Note also that there are no long-term trends of the averages.

\section{Solar wind dependencies}

To investigate the influence on the convection of various solar wind and IMF quantities, such as the solar wind dynamic pressure $P_{\text {dyn }}$, the solar wind electric field $E_{r, s w}$, the IMF 

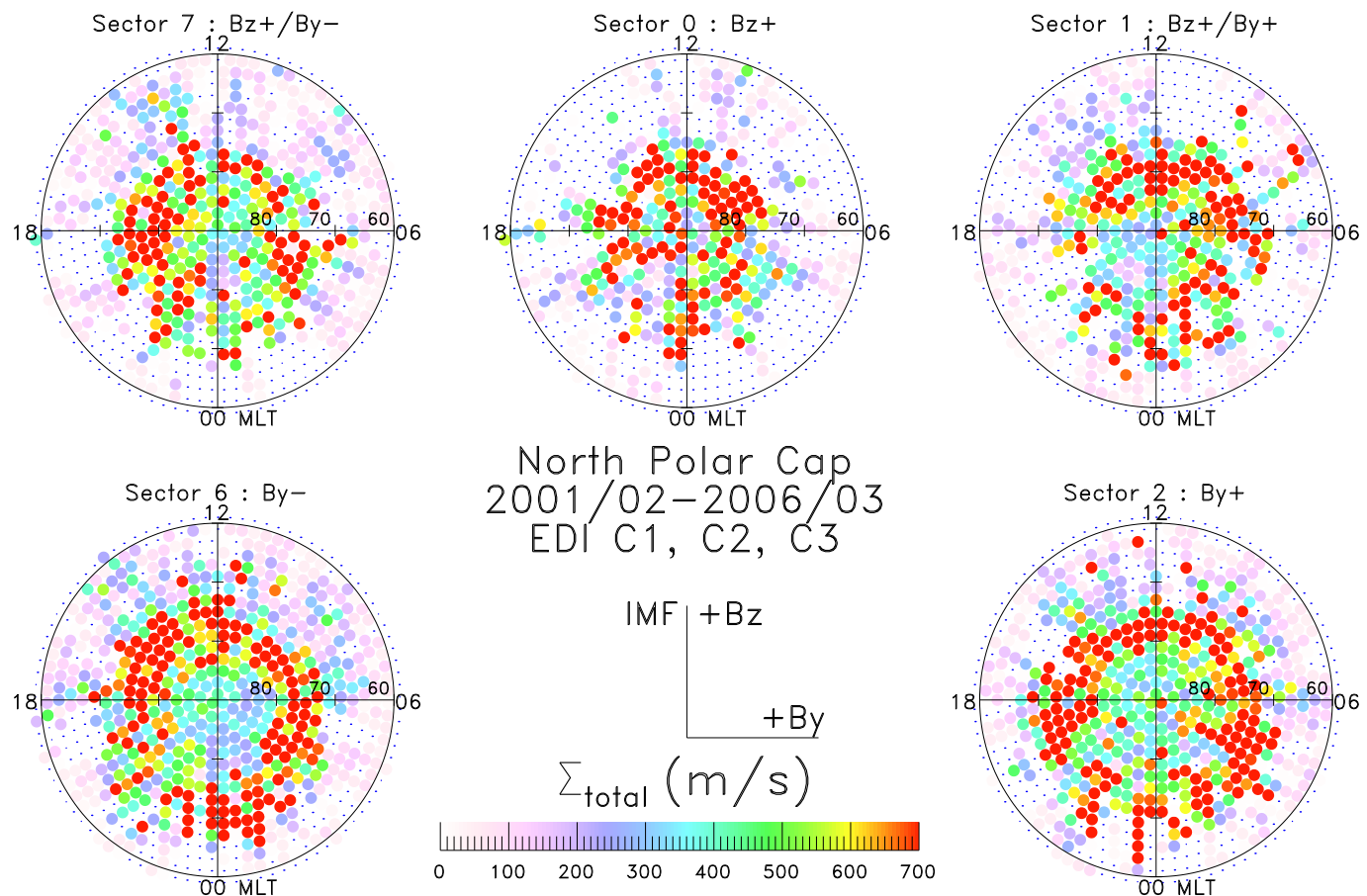

North Polar Cap
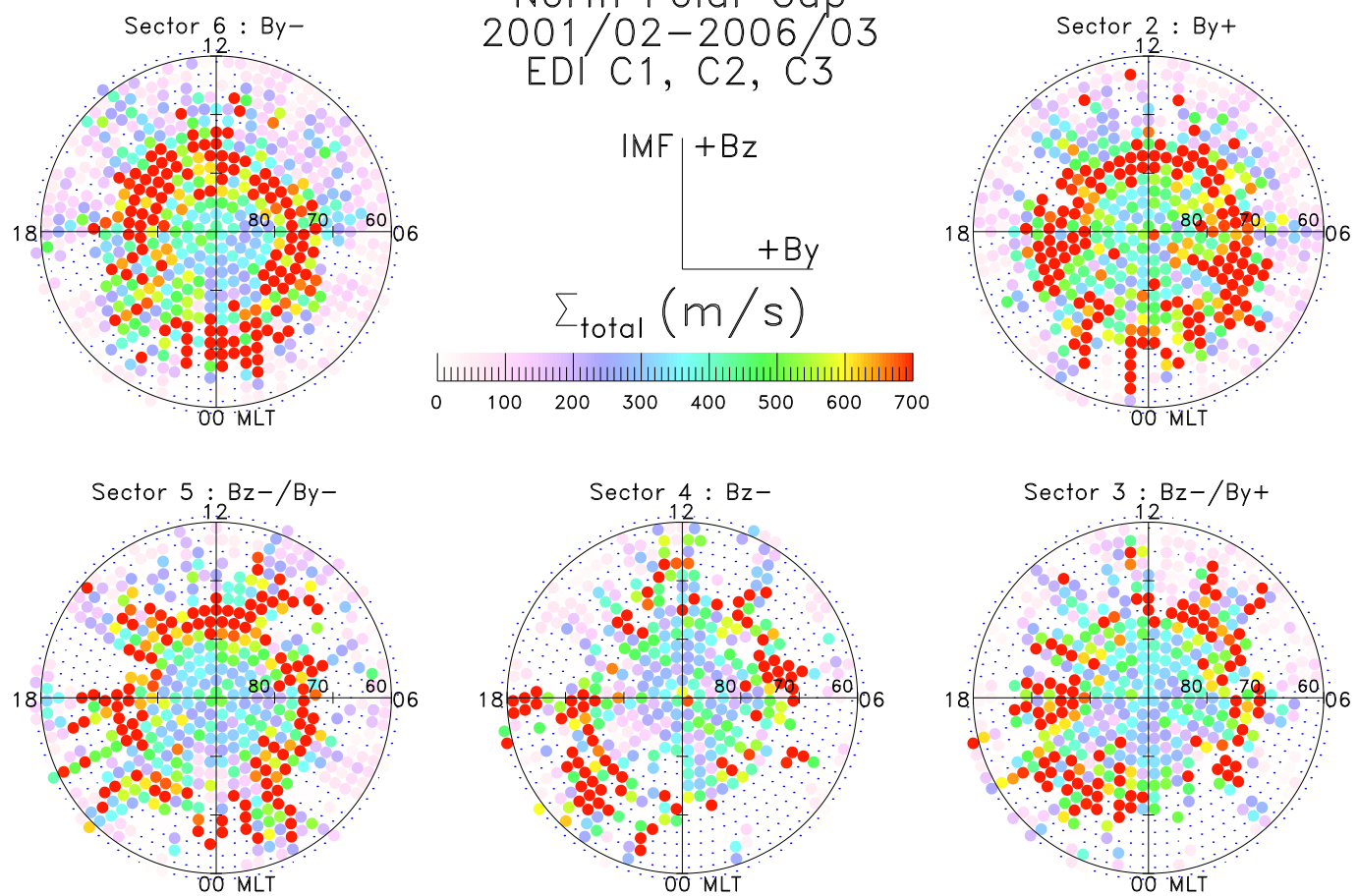

Fig. 5. Standard deviation of the total vector variation $\left[\mathrm{m} \mathrm{s}^{-1}\right]$ of EDI drift vectors mapped to the Northern high latitude ionosphere for the time interval from Feb 2001 to Mar 2006. As in Figs. 3 and 4, each circular panel shows magnetic local time (MLT) versus magnetic latitude with $60^{\circ}$ at the outer border. The color bar indicates the magnitude of the standard deviation, which is calculated for each bin individually, based on at least three valid mapped drift vectors within the bin.

magnitude $\left|B_{y z}^{\mathrm{IMF}}\right|$, and the $D_{s t}$ index, we bin our data set into a number of subsets for each of these parameters. The binning is a compromise between adequate resolution and sufficient data coverage. A good and homogeneous coverage is particularly important for the construction of the potential patterns. Large gaps in the longitudinal or latitudinal coverage cause problems with the Legendre polynomial expansion, which can lead to artifacts in the potential distribution (see Sect. 3.5 in Paper 1). For this reason, we calculate the average convection velocity within the central polar cap (magnetic latitude $\left|\phi_{m}\right|>80^{\circ}$ ) for each subset. Note that this selected area typically does not include the low latitude regions of convection return flow.
In the following, we use the magnitude of the average velocity components $\left\langle V_{x}\right\rangle$ and $\left\langle V_{y}\right\rangle$ as measures for the magnetospheric convection velocity across the center polar cap as well as $\langle V\rangle$, which represents the average of the velocity magnitudes. $V_{x}, V_{y}$ and $V_{z}$ are the mapped EDI drift velocity components at ionospheric level $(400 \mathrm{~km})$ in Solar Magnetic (SM) coordinates ( $V_{z}$ is usually very small in the high-latitude ionosphere). Note that the antisolar velocity is plotted with an inverted sign $\left(\left\langle-V_{x}\right\rangle\right)$ in the following figures of this section for better comparison with $\langle V\rangle$ dependencies.

\subsection{IMF direction}

Figure 7 shows the cross-polar cap potential (upper panel) as well as the average velocities defined above versus clock 


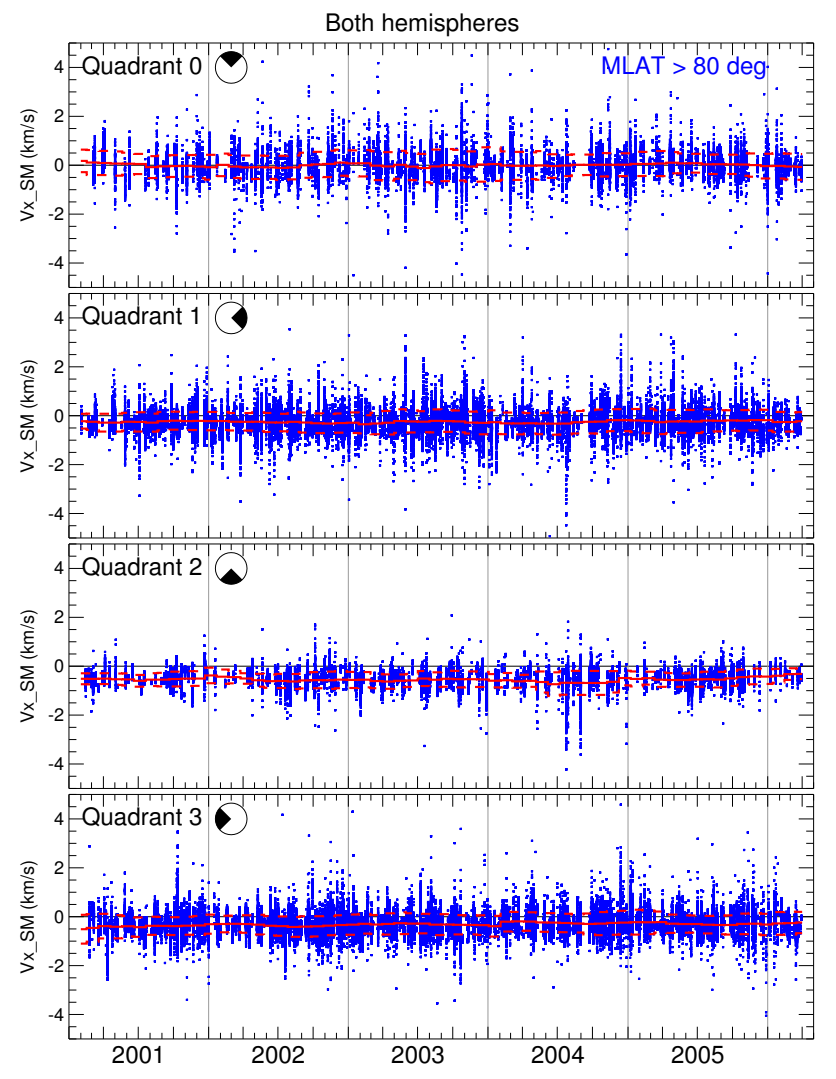

Fig. 6. 62 months of mapped EDI drift measurements in $\mathrm{X}_{S M}$ direction within the central polar cap region (magnetic latitude $\left|\phi_{m}\right|>80^{\circ}$ ), plotted as condensed time series for the four different quadrants of the IMF clock angle distribution. As indicated with the small circle inlet for each respective panel, quadrant 0 of clock angle distribution comprises $\pm 45^{\circ}$ centered around $0^{\circ}$ clock angle, quadrant 1 the same angular width centered around $90^{\circ}$, and so forth. Boxcar averages ( 6 month centered window) are shown as red lines and their standard deviations are indicated by the dashed red lines.

Table 2. Average values of the sliding boxcar mean values for 62 months of mapped EDI drift measurements in $\mathrm{X}_{S M}$ direction (red lines in Fig. 6) and their average standard deviations for each quadrant of the IMF clock angle distribution individually.

\begin{tabular}{cccc}
\hline Quadrant & $\begin{array}{c}\text { IMF } \\
\text { direction }\end{array}$ & $\begin{array}{c}\text { Mean value } \\
\mathrm{m} / \mathrm{s}\end{array}$ & $\begin{array}{c}\text { STDDEV } \\
\mathrm{m} / \mathrm{s}\end{array}$ \\
\hline 0 & $B_{z}+$ & +4 & 506 \\
1 & $B_{y}+$ & -252 & 420 \\
2 & $B_{z}-$ & -544 & 308 \\
3 & $B_{y}-$ & -317 & 414 \\
\hline
\end{tabular}

angle in 16 steps of $22.5^{\circ}$ each (middle panel) and the corresponding spatial coverage (lower panel). This figure complements Figs. 7 and 8 in Paper 1, but has twice the resolution in clockangle. The cross-polar cap potential is derived from potential pattern that were constructed with all data points of the
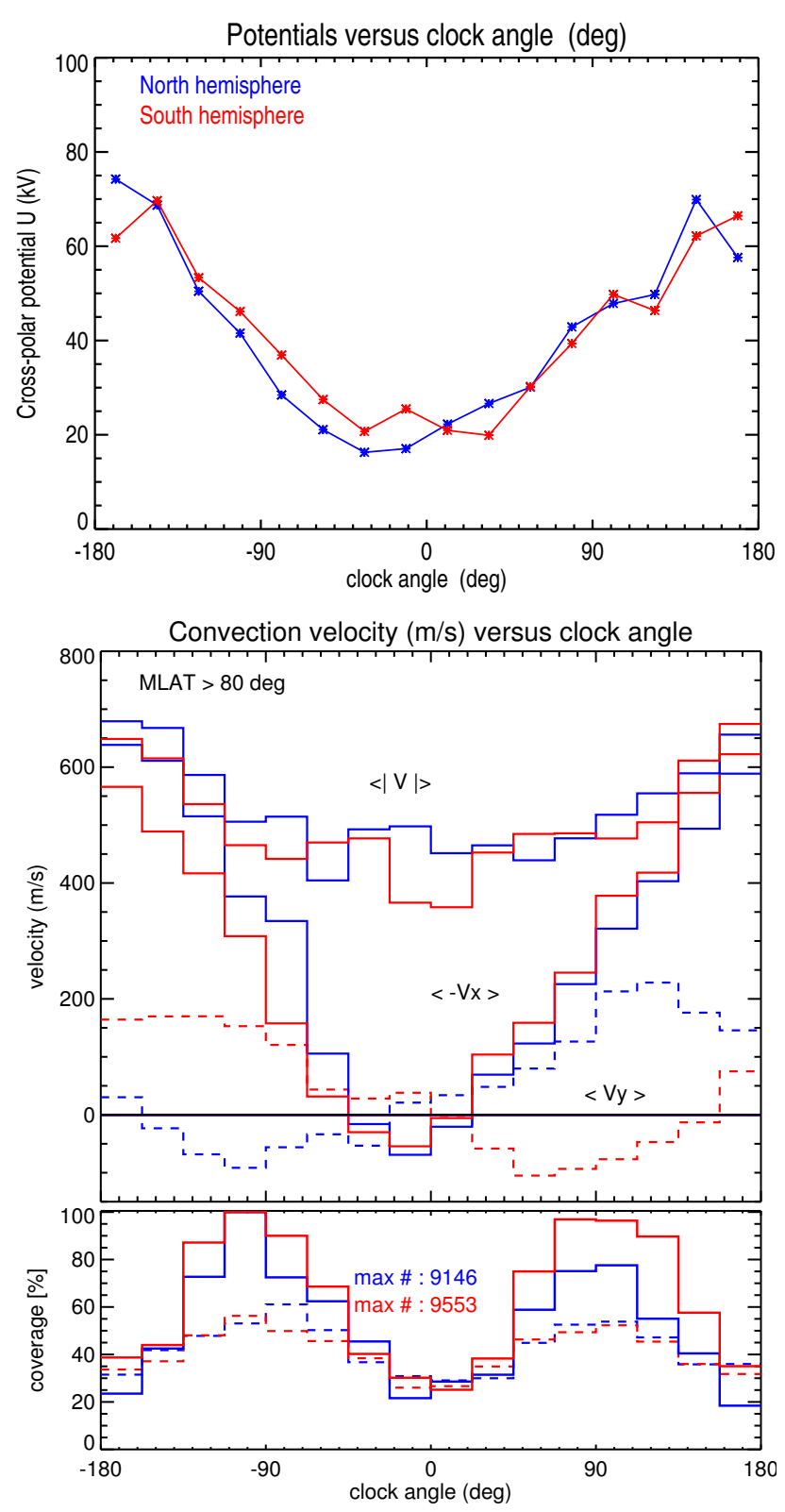

Fig. 7. Top: Polar cap potentials as a function of clock angle for the Northern (blue) and Southern (red) Hemisphere. The clock angle range is divided in 16 steps of equal width $\left(22.5^{\circ}\right)$. The potentials in this panel are derived from the full high-latitude $\left(>58^{\circ}\right)$ convection pattern for each bin, as explained in Paper 1. Middle: The average convection velocities $\langle V\rangle,\left\langle-V_{x}\right\rangle$, and $\left\langle V_{y}\right\rangle$ (dashed) within the central polar cap at magnetic latitudes $\left|\phi_{m}\right|>80^{\circ}$ as function of clock angle, using the same colour coding and bin-step widths as above. Bottom: coverage characteristics for each bin individually. The solid lines, which refer to the middle panel, show the relative number of data points within the high-latitude circle $\left(>80^{\circ}\right)$ for each bin, as percentage of the maximum number noted within the panel. The dashed lines refer to the upper panel, indicating the percentage of valid grid points of the full area of the polar cap potential pattern construction. 
bin-step interval as explained in Sect. 3.5 of Paper 1. The potential difference between the maximum and minimum value of the potential pattern is used for the curves in the upper panel. In most cases, this co-incides with the cross-polar cap potential difference between the main cells, the positive focus of the dawn cell and the negative of the dusk cell.

The figure confirms the rough mirror symmetry between North and South as well as the remaining minor hemispheric differences. There are different minimum positions of the potentials for North and South close to zero degree clock angle $\left(B_{z}^{\mathrm{IMF}}+\right)$ in the upper panel. The Northern Hemisphere shows a smooth broad minimum slightly shifted toward negative clock angles while at the Southern Hemisphere this minimum near $0^{\circ}$ clock angle is less clear and seems rather shifted toward positive values.

At southerly clock angles the two curves of $\langle V\rangle$ and the two curves $\left\langle-V_{x}\right\rangle$ in the middle panel of Fig. 7 are close, which means that the velocity directions are rather steady in antisolar direction over the central polar cap. This indicates that the contribution of $\left\langle V_{y}\right\rangle$ (dashed), being approximately mirror symmetric between North and South with respect to $0^{\circ}$ clock angle, is negligible. By contrast, at northerly clockangles the $\langle V\rangle$ and $\left\langle-V_{x}\right\rangle$ curves are quite far apart and $\left\langle V_{y}\right\rangle$ is comparable to $\left\langle V_{x}\right\rangle$, indicating that the directions are less steady: averaging the magnitudes then gives a large value for $\langle V\rangle$, while the variable directions causes partial compensation in the individual components. $\left\langle V_{x}\right\rangle$ even becomes positive on average for values near $0^{\circ}$ clock angle (northward IMF). This is due to the appearance of lobe cells with sunward convection at high latitudes. The minimum value of $\left\langle-V_{x}\right\rangle$ is slightly shifted toward negative clock angles for both Northern and Southern Hemisphere. This behaviour is similar to the potential variation in the upper panel resulting in steeper variation in the range of negative clock angles compared to the positive half-sphere.

The averaged convection velocity (middle panel of Fig. 7) reveals some North-South asymmetry, particularly in $\langle V\rangle$ and $\left\langle-V_{x}\right\rangle$ (when mirrored the southern curve at $0^{\circ}$ clock angle). This is presumably partially a result of the conductivity differences discussed in Sect. 2, but mainly the result of the mirror-symmetric action of the $B_{y}^{\mathrm{IMF}}$ dependence. Deviations of the geomagnetic field configuration from symmetry, in particular at ionospheric altitudes, will probably also affect the results (see also the discussion later in Sect. 4.5).

\subsection{Effect of IMF magnitude $\left|B_{y z}^{\mathrm{IMF}}\right|$}

In a recent paper, Ruohoniemi and Greenwald (2005), examined the influence of the IMF magnitude in the GSM y$\mathrm{z}$ plane $\left(\left|B_{y z}^{\mathrm{IMF}}\right|\right)$ on the convection patterns. They divided the IMF magnitude $\left|B_{y z}^{\mathrm{IMF}}\right|$ into three bins: $0-3 \mathrm{nT}, 3-5 \mathrm{nT}$ and 5-10 nT, and found that higher values of $\left|B_{y z}^{\mathrm{IMF}}\right|$ correspond to higher polar cap potentials. The only exception was found for pure northward IMF, where the polar cap potential remained fairly low and stable. For northward IMF values
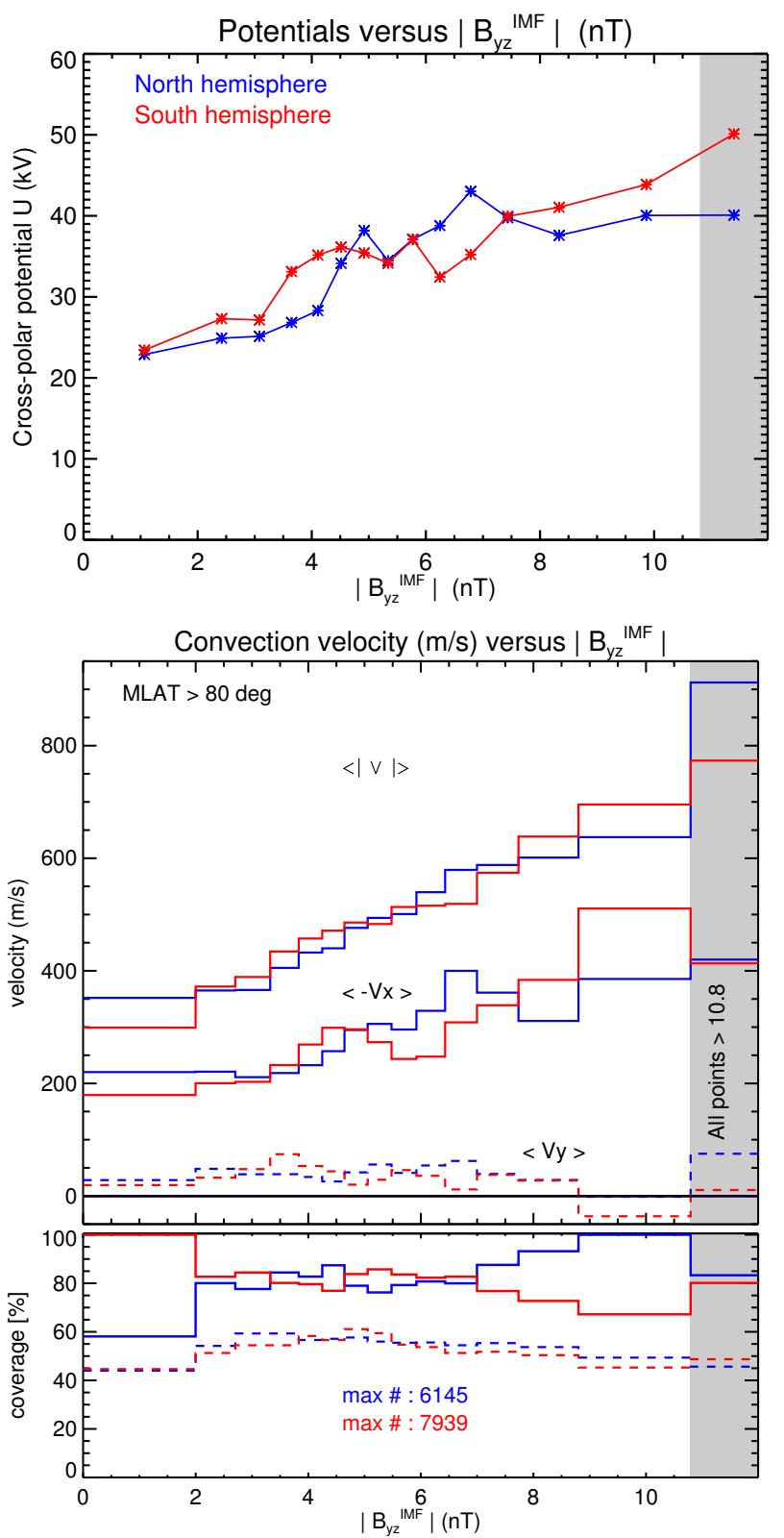

Fig. 8. Top: Polar cap potentials derived from the full high-latitude convection pattern for each bin as a function of $\left|B_{y z}^{\mathrm{IMF}}\right|$ for the Northern (blue) and the Southern Hemisphere (red). Middle panel: Average velocities as a function of the IMF magnitude $\left|B_{y z}^{\mathrm{IMF}}\right|$ within the central polar cap at magnetic latitudes $\left|\phi_{m}\right|>80^{\circ}$ only. Lower panel: coverage for each bin as explained in Fig. 7, except that we now used variable bin widths so that the amount of data points are about equal in each bin-step.

in the 5-10 nT range, they also found signatures of sunward convection and the emergence of lobe cells in the high latitude dayside polar cap. $\left|B_{y z}^{\mathrm{IMF}}\right|$ is one of the parameters that determines the solar wind electric field and is thus expected to influence the cross polar cap potential. 
To perform a similar analysis, we have combined all IMF directions, but divided the parameter range into more bins. These bin-steps were chosen such that they contain about the same amount of data - the steps are therefore not equidistant in contrast to the previous figure. The number of the steps was chosen such that the number of data points in each bin is sufficient for the velocity averages, and guarantees at the same time a good grid point coverage for the polar cap potential pattern, which results in 15 non-equal steps. The resulting coverage is illustrated in the bottom panel of Fig. 8. The full lines show the relative number of data points in each bin as percentage of the maximum number for the Northern and Southern Hemispheres separately. The deviations from the maximum number and the varying relative coverage between North and South is due to small hemispheric differences. The dashed lines show the percentage of valid grid points within the full area for the polar cap potential pattern construction (see Paper 1, Sect. 3.5) which is used for the upper panel.

The upper panel of Fig. 8 shows the derived cross-polar cap potential as a function of $\left|B_{y z}^{\mathrm{IMF}}\right|$ for both the Northern (blue line) and Southern Hemisphere (red line). They show the same positive trend, although there are some non-regular variations in the middle range from about $3 \mathrm{nT}$ to $7 \mathrm{nT}$. For small magnitudes of $\left|B_{y z}^{\mathrm{IMF}}\right| \lesssim 3 \mathrm{nT}$, the cross-polar cap potential has a minimum value between about $20 \mathrm{kV}$ to $25 \mathrm{kV}$. Up to about $10 \mathrm{nT}$, there is a positive correlation, in agreement with Ruohoniemi and Greenwald (2005). For $\left|B_{y z}^{\mathrm{IMF}}\right|$ values above the range shown in Fig. 8, the potential construction suffers from poor spatial coverage. The last step, marked with grey shading, contains therefore all data points above $\approx 10.8 \mathrm{nT}$.

The middle panel of Fig. 8 shows the dependence on $\left|B_{y z}^{\mathrm{IMF}}\right|$ of the convection velocities $\langle V\rangle,\left\langle-V_{x}\right\rangle$, and $\left\langle V_{y}\right\rangle$. As already mentioned above, these averages are derived with data points from the central polar cap area (at $\left|\phi_{m}\right|>80^{\circ}$ ). The binning of the whole parameter range is the same as for the potential pattern in the upper panel. The velocities in the middle panel, particularly $\left\langle-V_{x}\right\rangle$, show approximately the same dependence on $\left|B_{y z}^{\mathrm{IMF}}\right|$ as the cross-polar cap potential in the upper panel, including the minor variations in the middle range. It can therefore be regarded to some extent as a proxy for the cross-polar cap potential. This will be utilized further in subsequent sections. The proxy character is not surprising given that $\boldsymbol{v}=\boldsymbol{E} \times \boldsymbol{B} / B^{2}$ and $\boldsymbol{B}$ is essentially constant within the auroral oval. While $\left\langle-V_{x}\right\rangle$ represents the localized dawn-dusk electric field strength within the central polar cap, the polar cap potential stands for the larger-scale, integrating effect of the solar wind-magnetosphere coupling.

Starting from a level of $\approx 200 \mathrm{~m} \mathrm{~s}^{-1}$ for the 2-3 lowermost bins, $\left\langle-V_{x}\right\rangle$ rises up to a level of about $400 \mathrm{~m} \mathrm{~s}^{-1}$ for the highest bins. At that end, the averages start to fluctuate about this level. This can be interpreted as an indication for saturation at $\left|B_{y z}^{\mathrm{IMF}}\right| \gtrsim 11 \mathrm{nT}$.
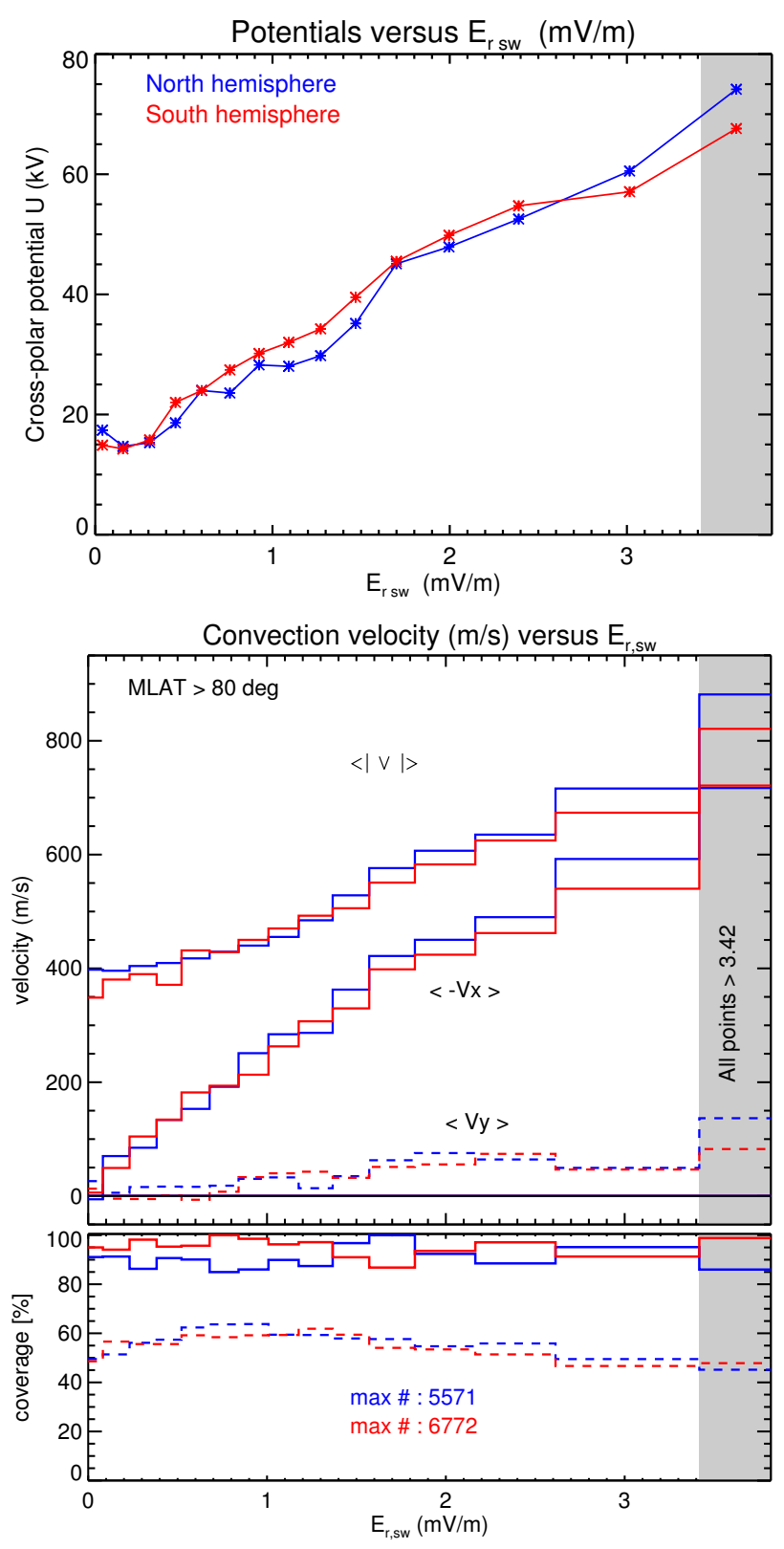

Fig. 9. Top: Cross-polar cap potentials as function of the solar wind reconnection electric field, $E_{r, s w}$, for both North (blue) and South (red) high-latitude convection pattern, derived for each bin separately (cf. Paper 1). Here, again we use variable step widths as in Fig. 8. Middle: Central polar cap convection velocities for the same variable bin steps as above, but averaged within the central polar cap at magnetic latitudes at $\left|\phi_{m}\right|>80^{\circ}$ only. The bottom panel shows the coverage characteristics for each bin like in Fig. 7.

\subsection{Dependence on solar wind electric field}

Figure 9 shows the cross polar cap potential estimations (upper panel), the average convection velocities $\left\langle-V_{x}\right\rangle$ and $\left\langle V_{y}\right\rangle$ $\left(>80^{\circ}\right)$, and the convection magnitude $\langle V\rangle$ (middle panel) as function of the solar wind electric field, projected along 

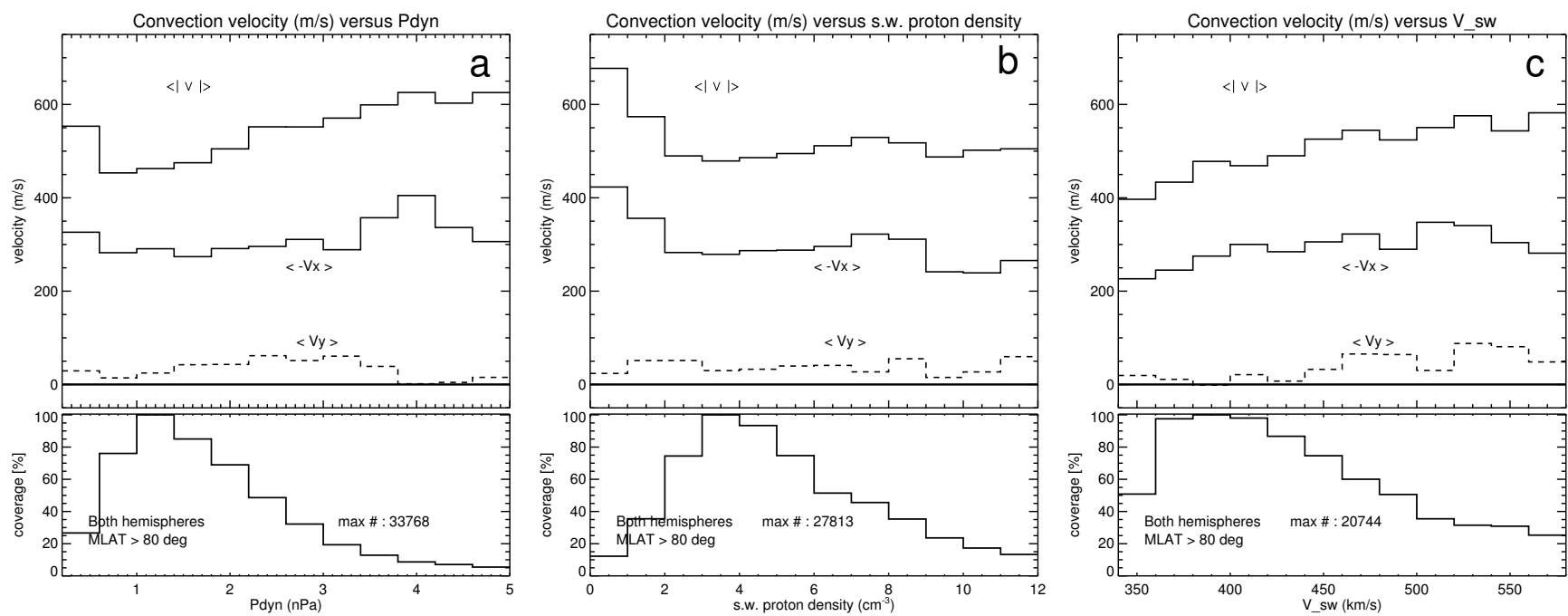

Fig. 10. (a): The polar cap convection velocity shown as function of the solar wind dynamic pressure $P_{\text {dyn }}$; (b, c): the corresponding dependencies of the solar wind proton density $n_{p}$ and velocity $\left|V_{s . w .}\right|$. Data points from both hemispheres have been combined for the analysis. The bottom panels indicate the spatial coverage characteristics for each bin within the high-latitude area $\left(\left|\phi_{m}\right|>80^{\circ}\right)$.

the dayside reconnection $\mathrm{X}$-line according to the method by Sonnerup (1974):

$E_{r, s w}=E_{y z, s w} \sin ^{2}(\theta / 2)=V_{x, s w} B_{y z, s w}^{\mathrm{IMF}} \sin ^{2}(\theta / 2)$

where $E_{y z, s w}$ is the solar wind electric field, $V_{x, s w}$ is the Xcomponent of the solar wind velocity, $\left|B_{y z}^{\mathrm{IMF}}\right|$ is the interplanetary magnetic field in the GSM yz-plane, and $\theta$ is the IMF clock angle. As seen from Eq. (3), this parameter strongly depends on the direction of the IMF. The electric field $E_{r, s w}$ serves as an indicator of the reconnection rate at the frontside magnetopause (dayside reconnection), or, for a fixed length of the reconnection line, of the cross-polar cap potential drop.

As expected, there is a clear correlation between the antisolar convection velocity $\left\langle-V_{x}\right\rangle$ and $E_{r, s w}$. Higher reconnection rates at the magnetopause cause faster transport of flux across the polar cap. As noted in Paper 1, the convection between the main cells is slow, but still antisunward over the central polar cap, for low values of $E_{r, s w}$ (which typically indicate a northward directed IMF). Closer inspection of the individually averaged components $\left\langle V_{x}\right\rangle$ and $\left\langle V_{y}\right\rangle$ show, that at low values of $E_{r, s w}$ the sunward convection, which is taking place within the lobe cells, and the usual antisunward convection cancel each other within the high-latitude circle at magnetic latitudes $>80^{\circ}$, so that $\left\langle-V_{x}\right\rangle$ shows the straight linear behaviour, starting at zero. In contrast, the potential curves have a finite magnitude of $\approx 15 \mathrm{kV}$ for vanishing $E_{r, s w}$ as they result from the remaining main convection cells. The $\left\langle V_{y}\right\rangle$ component increases slowly with increasing values of $E_{r, s w}$, starting close to zero and raising up to $50-100 \mathrm{~m} \mathrm{~s}^{-1}$ over the range shown in Fig. 9. While there is linear correlation for values of $E_{r, s w}$ (up to almost $2 \mathrm{mV} \mathrm{m}^{-1}$ ), the curve then flattens out, but does not saturate, as demonstrated by the fact that the last bin, which collects all $E_{r, s w}$ values above approximately $3.4 \mathrm{mV} \mathrm{m}^{-1}$, still shows a significant increase. This issue of potential saturation has been addressed by Siscoe et al. (e.g. 2002); Hairston et al. (e.g. 2005).

\subsection{Dependence on solar wind dynamic pressure}

As mentioned, due to the nature of statistical studies, we are not able to investigate the direct response to time variances such as pressure pulses or sudden reductions in the dynamic pressure. But we can draw conclusion about the statistical behaviour of a large data set.

Figures $10 \mathrm{a}-\mathrm{c}$ show the solar wind dynamic pressure $P_{\text {dyn }}$ (left panel) together with the parameters it is constructed from, namely the solar wind proton density $n_{p}$ (middle panel) and the solar wind velocity $\left|V_{s w . w}\right|$ (right panel). They are related according to $P_{\mathrm{dyn}}=m_{p} n_{p} V_{s w}^{2}$, where $m_{p}$ is the proton mass. No significant North-South asymmetries were found for the convection velocities. Therefore we are combining measurements from both hemispheres in this plot. This is done by folding the Southern data points into the Northern by inverting the $B_{y}^{\mathrm{IMF}}$ dependence. The bottom panels indicate the spatial coverage characteristics for each bin within the high-latitude area $\left(\left|\phi_{m}\right|>80^{\circ}\right)$.

The drift velocity averages show a weak positive correlation with $P_{\text {dyn }}$ (upper panel of Fig. 10a). Even more interestingly, at very low pressures there seems to be a significant peak, that is, low $P_{\text {dyn }}$ are related to higher convection velocities. The reason for this is not clear, but the data coverage is good, so the effect seems to be real and it is related to the solar wind density, which reveals this peak at very low densities (upper panel of Fig. 10b). The further variation with the proton density is quite flat, except of a secondary small peak 

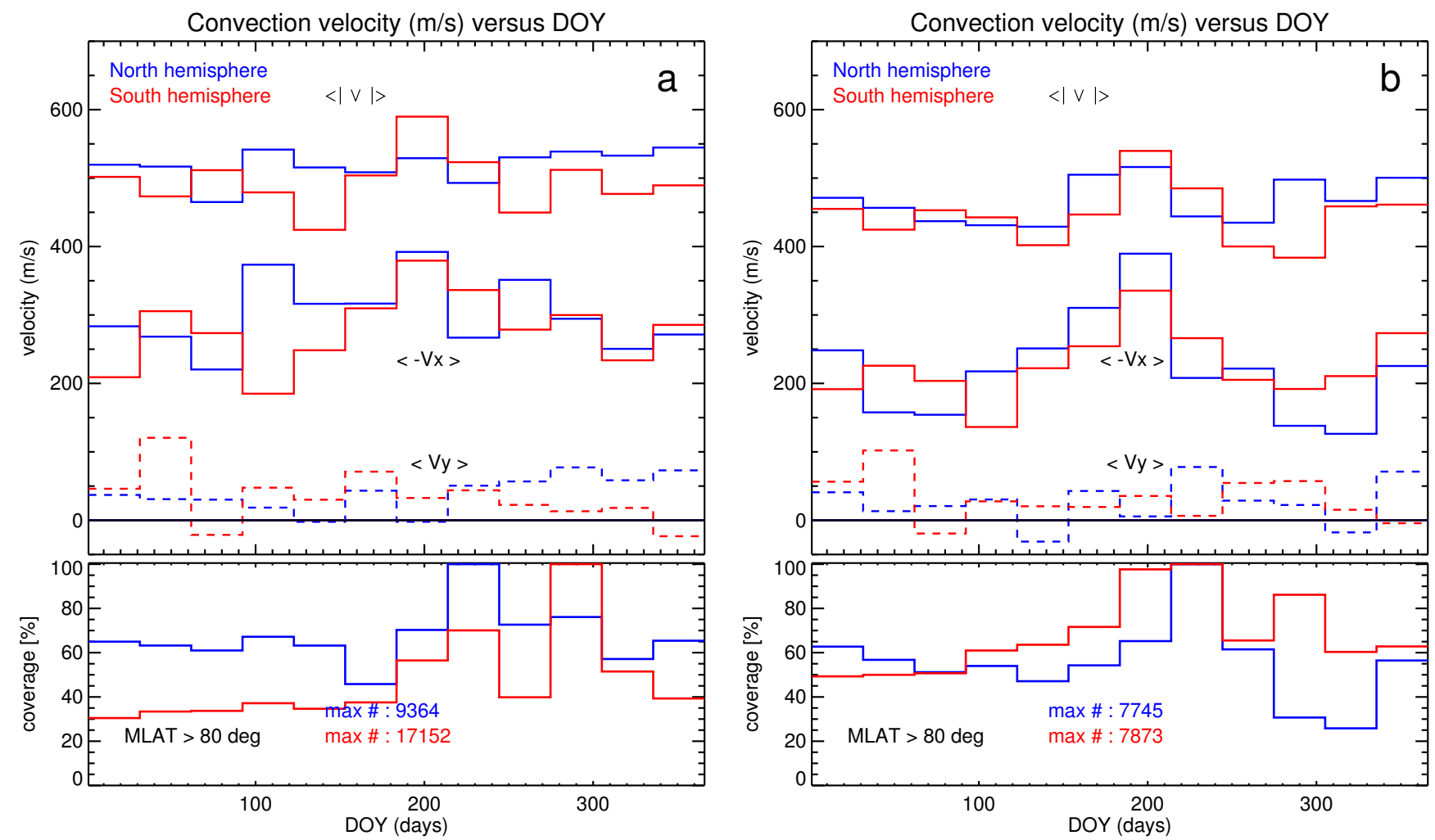

Fig. 11. Average convection velocity variations versus Day of the Year (DOY) (or seasonal dependencies) for the Northern (blue curves) and Southern Hemisphere (red). Left (a): using all data points of our data set. Right (b): using a filtered data set with $D_{s t}>-30 \mathrm{nT}$ only (quiet conditions). The bottom panels illustrate the data coverage of the 12 equally sized steps corresponding to approximately monthly resolution.

in the range of $7-8 \mathrm{~cm}^{-3}$. The slow rise of the convection velocities with increasing $P_{\text {dyn }}$ is explained by a corresponding rise in the solar wind speed, shown in Fig. 10c.

Equating the velocity variations with cross-polar cap potential variations, we expect that there should be an increased potential at very low $P_{\text {dyn }}$ values which is due primarily to a solar wind density effect. The amplitude of this effect is up to about $25 \%$ relative to the level at mean solar wind dynamic pressure.

\subsection{Seasonal variations}

In Fig. 11 we tried to estimate possible seasonal variations that could be revealed from our data set. Due to the orbital constraints, as explained in Paper 1, Sect. 3.4, only the very central part of the polar cap can be considered as equally covered with data points throughout the whole year, though the mapped drift vectors might originate from different spatial volumes of the magnetosphere.

Several studies in the past investigated the seasonal dependencies of the large-scale high-latitude convection pattern, but many studies (e.g., De La Beaujardiere et al., 1991; Rich and Hairston, 1994; Weimer, 1995; Ruohoniemi and Greenwald, 1995; Milan et al., 2001) came to different or even con- tradictory results. Ruohoniemi and Greenwald (1995, 2005) found that the seasonal effect is similar to that of the sign of $B_{y}^{\mathrm{IMF}}$. They state that the combination of $\mathrm{B}_{y}+$ /summer ( $\mathrm{B}_{y}-/$ winter) reinforces the tendency of the $B_{y}^{\mathrm{IMF}}$ sign factor to sculpt the dusk and dawn cells into more round/crescent shapes and to shift the crescent cell across the midnight MLT meridian while lowering the total cross polar cap potentials. The non-reinforcing combinations, on the other hand, produce elevated cross-polar potentials, especially for $\mathrm{B}_{y}-$ /summer conditions and they found an overall tendency for the cross polar cap potential to increase from winter to summer. These conclusions of the Ruohoniemi and Greenwald (2005) study are based on Northern Hemisphere observations of the SuperDARN network.

The upper pair of curves in the upper panel of Fig. 11a, showing the seasonal variation of the averages of the drift magnitudes, $\langle|V|\rangle$, reveal the generally higher level of Northern Hemisphere velocity. On average, this amounts to about $7 \%$ for the whole area of our high-latitude grids, as already mentioned in Sect. 1, and it is slightly smaller but still visible within the $80^{\circ}$ circle shown here. The relative seasonal variations of this parameter are very small, i.e., less than $10 \%$ at the Northern Hemisphere and somewhat larger (up to $\approx 20 \%$ ) 
at the Southern. The weak trend shows slight maxima (if ever) during winter months of the respective Hemisphere. The velocity averages $\left\langle-V_{x}\right\rangle$ in Fig. 11a show a somewhat different behaviour. The relative seasonal variations and the variations from month to month are larger and the annual variation seem to be more-or-less in phase with the upper curves in case of the Southern Hemisphere, while it seems to be in opposite phase at the Northern.

After filtering the time series for geomagnetically undisturbed conditions, as is done in Fig. $11 \mathrm{~b}$ for $D_{s t}>30 \mathrm{nT}$, a clear semiannual variation becomes evident with larger convection values during solstices for both hemispheres. The July maximum (Northern summer) seems to be more pronounced than that of December/January. It is well known that geomagnetic storms are more frequent during the equinoxes. This was discovered by analyzing time series of geomagnetic indices. The semiannual variation (and to a minor degree also a diurnal variation) has been explained by the Russell-McPherron (R-M) effect (Russell and McPherron, 1973) as being due to the geometrical relationship between the Earth's dipole axis and the Parker spiral plane. Additionally, the semiannual effect has recently be shown to originate also from the periodical change of the solar wind-magnetosphere-ionosphere (SMI) coupling efficiency (e.g., Cliver et al., 2004; Nagatsuma, 2006) which leads to more frequent and stronger geomagnetic storms near the equinoxes. During quiet-time conditions, on the other hand, we observe on average enhanced and more ordered convection during solstice periods, as shown in Fig. 11b. The reason for this is not yet clear, but can probably be related to thermosphere-ionosphere influences on the magnetospheric convection. This should be analyzed in more detail in a future study.

The relative variations for unfiltered data (Fig. 11a) are generally larger at the Southern Hemisphere. Seasonal variations are likely to be related to different illumination conditions (cf. Fig. 2) and therefore different ionospheric conductances. The larger relative variations in the Southern Hemisphere might also partially be caused by differences in the geomagnetic field configuration. Whereas the Northern Hemisphere IGRF field poleward of $58^{\circ}$ is fairly homogeneous, the Southern Hemisphere contains many crustal anomalies, causing a larger spatial variation in the magnetic field. Values of the IGRF magnetic field at $400 \mathrm{~km}$ altitude in the Northern Hemisphere $\geq 58^{\circ}$ range from 42.9 to $49.9 \mu T$, with an average of $47.4 \mu T$. For the Southern Hemisphere, the corresponding spatial variation is between 30.8 to $54.7 \mu T$, with an average of $49.7 \mu T$. Time variations of the IGRF field in the period from February 2001 to March 2006 are slow and insignificant in this connection.

The data coverage, shown in the bottom panel of Fig. 11, is largest around the September equinox for both hemispheres with the Southern maximum being larger than the Northern. During this time of the year, the Cluster satellites have their apogee in the tail magnetosphere and spend relatively long

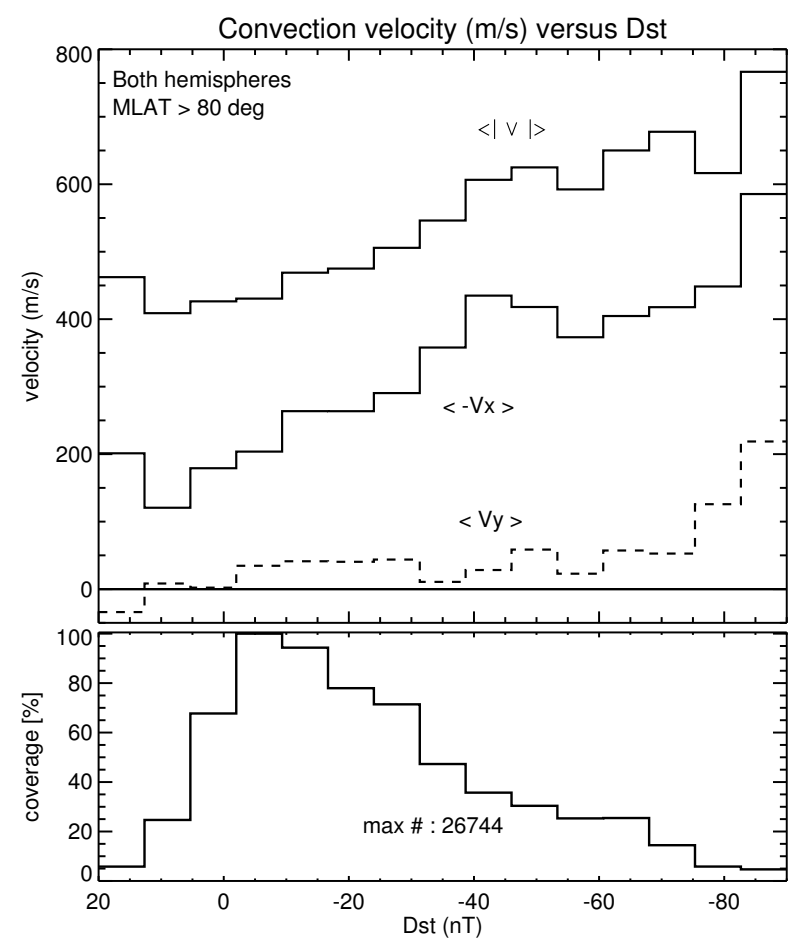

Fig. 12. Top: Dependence of the average convection velocities on the $D_{s t}$ index, with data from both hemispheres combined. Bottom: number of data points with equally sized steps, in percentage of the maximum indicated.

time intervals there. As mentioned earlier, due to the southward tilt of the line of apsides of the Cluster orbit, the satellites spend more time below the neutral sheet on the magnetospheric tailside which maps to the Southern Hemisphere nightside ionosphere.

Generally, the seasonal effect is minor with maximum amplitudes of $10 \%$ to $30 \%$ at most in the cross-polar potential as deduced from the locally confined average velocity estimations of $\left\langle-V_{x}\right\rangle$ within the central polar cap. According to the same reasoning, an overall tendency for a semiannual variation of the cross polar cap potential during geomagnetically undisturbed conditions is likely with on average larger potentials during solstices.

\subsection{Dependence on the $D_{s t}$ index}

Figure 12 shows the correlation with the Disturbed Storm Time $\left(D_{s t}\right)$ index. The $D_{s t}$ index is a measure of the horizontal magnetic deflection on the Earth at equatorial latitudes. Negative deflections in $D_{s t}$ are mainly controlled by Earth's ring current, though the solar wind pressure also contributes (e.g., Siscoe et al., 1968; Burton et al., 1975; O'Brien and McPherron, 2000). Positive deflections are usually caused by pressure enhancements in the solar wind which cause a displacement of the magnetospheric drift shells. 


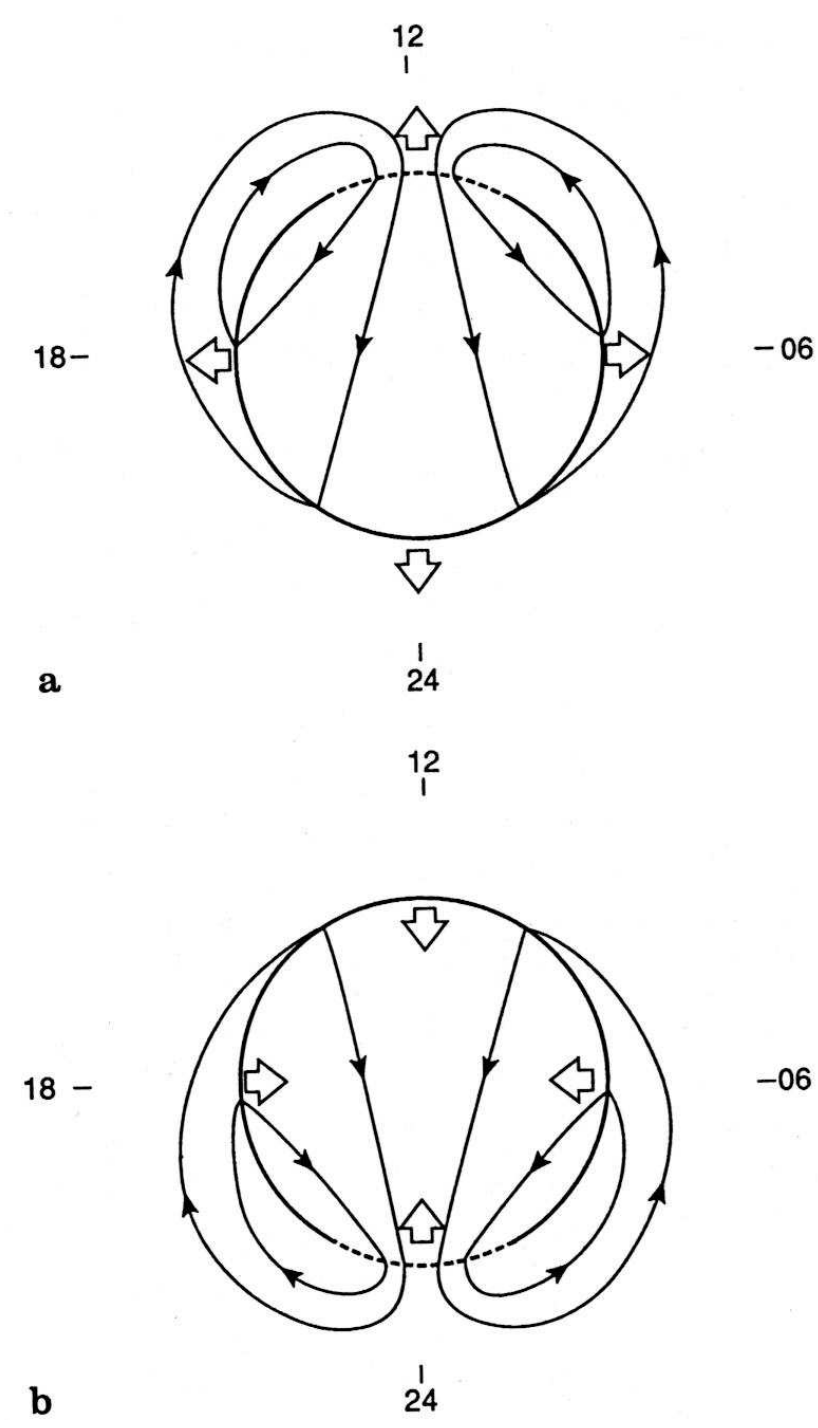

Fig. 13. This sketch, taken from Cowley and Lockwood (1992), illustrates the polar cap convection for (a) dayside reconnection not balanced by concurrent nightside reconnection; (b) tail reconnection without concurrent dayside reconnection. For further details see Cowley and Lockwood (1992).

Typically, the ring current increases (thus creating a negative deflection of $D_{s t}$ ) during periods of increased cross-tail electric field, which is typically caused by enhanced dayside reconnection. From this argument, one should therefore expect some degree of correlation with polar cap convection velocity. However, processes in the magnetotail, such as bursty bulk flows or substorm activity also influence the $D_{s t}$ index (e.g., Baumjohann et al., 1996; Friedrich et al., 1999; Baker et al., 2001). Energization is usually very fast, whereas various loss processes such as charge exchange, pitch angle scattering, radial diffusion etc. have much longer time scales (e.g., Cowley, 1977; Daglis et al., 1999).
The clear correlation apparent from Fig. 12 supports the idea that enhanced convection translates into an increase in the cross tail electric field, and thus an energization of the ring current and subsequent negative $D_{s t}$ deflection. Similarly, the higher convection velocity for positive $D_{s t}$ values may reflect the solar wind pressure pulses and associated displacements of magnetospheric drift shells. No North-South asymmetries were found, so the convection velocities are averages based on measurements from both hemispheres.

\section{Effect of tail magnetic activity}

High-latitude plasma convection is not completely determined by dayside coupling, as expressed by the concurrent orientation of the IMF at the magnetopause, but also by processes in the magnetotail that are only indirectly linked to concurrent IMF conditions (e.g., Cowley and Lockwood, 1992) as schematically illustrated in Fig. 13. We demonstrate this point with the EDI potential maps for northward IMF. This is because for strongly northward IMF, no dayside reconnection is expected to occur, and thus the patterns is most sensitive to effects from tail activity.

To enhance the statistical relevance, we use instead of Sector 0 the full "Quadrant 0" of northward directed IMF, i.e. a clock angle range of $\pm 45^{\circ}$ centered around $0^{\circ}$, as well as both hemispheres together (mirroring $B_{y}^{\mathrm{IMF}}$ of the Southern high-latitude data points by sign change). Dividing the Quadrant 0 data according to the level of the ASYM-H index that we have taken as proxy for magnetic activity, and constructing the corresponding potential maps, as described in Paper 1, we arrive at Fig. 14. The potential maps for low ASYM-H (left panel) and high ASYM-H (right) are constructed from about equally populated half-distributions (see Fig. 1), below and above an ASYM-H value of $20 \mathrm{nT}$, respectively.

For the dayside, both maps show the lobe cells resulting from lobe reconnection, as expected for strongly northward IMF. But for the nightside, there is a strong difference in the spacing of the iso-potential contours, indicating much stronger convection for high values of ASYM-H. This is exactly what is expected from reconnection in the magnetotail associated with high magnetic activity, as shown in Fig. 13.

\section{Summary}

Based on a data set of more than $5800 \mathrm{~h}$ of convection velocity measurements from the Cluster EDI experiment, mapped into the ionosphere, we have investigated the variability as well as the solar wind and IMF dependencies of the highlatitude convection.

- The variability of the high-latitude convection shows, similar to the convection itself, characteristic spatial patterns of dependence of the $B_{y}^{\mathrm{IMF}}$ component which 

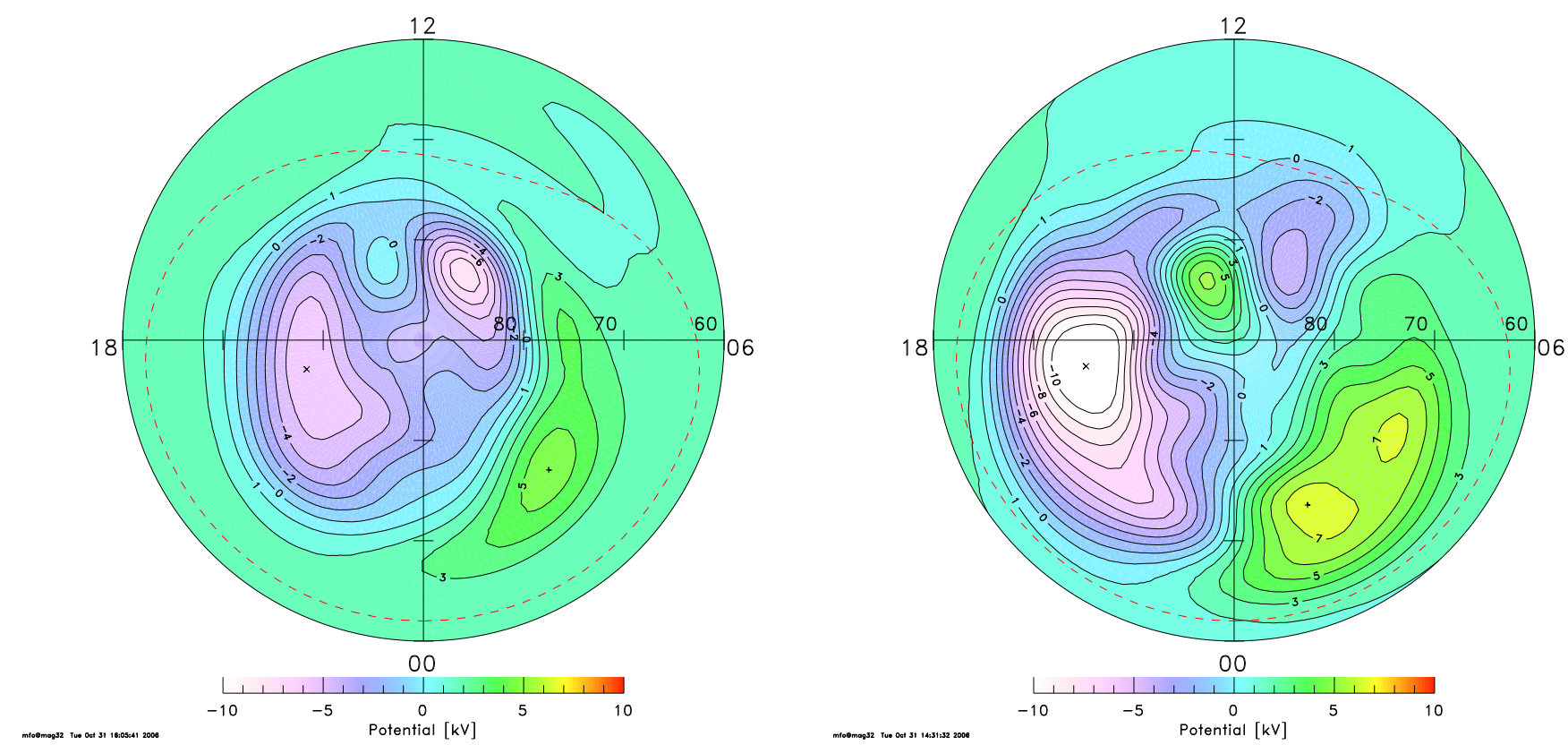

Fig. 14. Potential patterns for northward IMF and two levels of nighside activity. Left: Low values of ASYM-H $<20 \mathrm{nT}$, indicating little or no auroral activity. Right: High ASYM-H values ( $\geq 20 \mathrm{nT}$ ), indicating strong partial ring current activity. The full Quadrant 0 of clock angle distribution, i.e. $\pm 45^{\circ}$ centered around $0^{\circ}$, and North and South Hemisphere combined have been used to derive the potential maps.

are mirror symmetric between the Northern and Southern Hemispheres. The variances (standard deviations) are of the same order as or even larger than the convection magnitude within the auroral oval in particular.

- The average convection velocity in $\mathrm{X}_{S M}$ direction (i.e., the dawn-dusk electric field strength) within the central part of the polar cap $\left(>80^{\circ}\right)$ can be used alternatively to the cross-polar cap potential for the study of magnetospheric forcings and their dependencies on various solar wind and IMF parameters. It shows very similar variations, but represents rather solar wind-magnetosphere coupling conditions at mostly 'open' flux tubes within the central polar cap.

- Correlation with external drivers such as the solar wind dynamic pressure and IMF magnitude in the GSM y$\mathrm{z}$ plane, $\left|B_{y z}^{\mathrm{IMF}}\right|$, are in agreement with earlier results (Papitashvili and Rich, 2002; Ruohoniemi and Greenwald, 2005; Matsui et al., 2005). The qualitative behaviour with positive correlations between the velocity (and thus potential) and these drivers are also reproduced by model calculations (e.g., Weimer, 2001).

- Except for purely northward directed IMF, there is a clear correlation between the IMF magnitude, $\left|B_{y z}^{\mathrm{IMF}}\right|$, and convection velocities. Our data set contains a larger proportion sunlit observations from the Southern Hemisphere. Average convection velocities there are systematically somewhat lower than Northern Hemisphere val- ues. A positive correlation also exist between $\left|B_{y z}^{\mathrm{IMF}}\right|$ and the polar cap potential, but no North-South asymmetry was found in the absolute values of the potentials.

- Low to moderate values of the solar wind electric field are positively correlated with the convection velocity. For values of $E_{r, s w}$ above approximately $2 \mathrm{mV} \mathrm{m}^{-1}$ the potential does not rise linearly with $E_{r, s w}$, but seems to flatten out.

- A positive correlation is found between convection velocity and solar wind dynamic pressure $P_{\mathrm{dyn}}$, but additionally there appears an enhanced convection speed (cross polar cap potential) for very low values of $P_{\mathrm{dyn}}$. This latter peak is due to solar wind density effects, the reason for which are not yet clear.

- Seasonal variations are found to be of minor importance (of the order of $10 \%$ to $30 \%$ ). They are more pronounced and show a clear semiannual variation with maxima of convection during solstices for geomagnetically quiet periods only.

- There is a positive correlation between ring current energization (reflected by the $D_{s t}$ index) and the convection velocity. This demonstrates that a part of the ring current is directly driven by enhanced dayside reconnection.

- We also find a positive correlation between nighside activity/processes in the magnetotail (reflected by the 
ASYM-H index) and convection velocity. A large ASYM-H index corresponds to higher polar cap potential.

Since our data set is not continuous in time, we are unable to address statistical time dependencies such as response times or decay times. Such studies will have to be based on shorter, continuous time segments in the data set. This will be addressed in future publications.

Acknowledgements. Work at GeoForschungsZentrum (GFZ) Potsdam was supported by Deutsche Forschungsgemeinschaft (DFG). Work at the Max-Planck- Institut für extraterrestrische Physik was supported by Deutsches Zentrum für Luft- und Raumfahrt (DLR). Research at the University of Bergen was supported by the Norwegian Research Council. Work by U.S. investigators was supported in part by NASA grant NNG04GA46G. Parts of the data analysis were done with the QSAS science analysis system provided by the UK Cluster Science Centre (Imperial College London and Queen Mary, University of London) supported by PPARC UK. We thank the ACE SWEPAM and MAG instrument teams and the ACE Science Center for providing the ACE data, and the World Data Center for Geomagnetism, Kyoto, for providing the $D_{s t}$ and ASYM-H indices. We thank M. Chutter for his support of EDI data analysis and processing, and G. Leistner for providing the averaged EDI data.

Topical Editor I. A. Daglis thanks two anonymous referees for their help in evaluating this paper.

\section{References}

Axford, W. I. and Hines, C. O.: A unifying theory of high-latitude geophysical phenomena and geomagnetic storms, Can. J. Phys., 39, 1433-1464, 1961.

Baker, D. N., Turner, N. E., and Pulkkinen, T. I.: Energy transport and dissipation in the magnetosphere during geomagnetic storms, J. Atmos. Terr. Phys., 63, 421-429, doi:10.1016/ S1364-6826(00)00169-3, 2001.

Banks, P. M.: Magnetospheric processes and the behavior of the neutral atmosphere, Space Res., 12, 1051-1067, 1972.

Baumjohann, W., Kamide, Y., and Nakamura, R.: Storms, substorms and the near-Earth tail, J. Geomag. Geoelectr., 48, 177185, 1996.

Boyle, C. B., Reiff, P. H., and Hairston, M. R.: Empirical polar cap potentials, J. Geophys. Res., 102, 111-125, 1997.

Bristow, W. A., Greenwald, R. A., Shepherd, S. G., and Hughes, J. M.: On the observed variability of the crosspolar cap potential, J. Geophys. Res., 109, A02203, doi:10.1029/2003JA010206, 2004.

Burke, W. J., Kelley, M. C., Sagalyn, R. C., Smiddy, M., and Lai, S. T.: Polar cap electric field structures with a northward interplanetary magnetic field, Geophys. Res. Lett., 6, 21-24, 1979.

Burton, R. K., McPherron, R. L., and Russell, C. T.: An empirical relationship between interplanetary conditions and Dst, J. Geophys. Res., 80, 4204-4214, 1975.

Cliver, E. W., Svalgaard, L., and Ling, A. G.: Origins of the semiannual variation of geomagnetic activity in 1954 and 1996, Ann. Geophys., 22, 93-100, 2004, http://www.ann-geophys.net/22/93/2004/.
Codrescu, M. V., Fuller-Rowell, T. J., and Foster, J. C.: On the importance of E-field variability for Joule heating in the high-latitude thermosphere, Geophys. Res. Lett., 22, 2393-2396, 1995.

Codrescu, M. V., Fuller-Rowell, T. J., Foster, J. C., Holt, J. M., and Cariglia, S. J.: Electric field variability associated with the Millstone Hill electric field model, J. Geophys. Res., 105, 52655274, 2000.

Cole, K. D.: Damping of magnetospheric motions by the ionosphere, J. Geophys. Res., 68, 3231-3235, 1963.

Coroniti, F. V. and Kennel, C. F.: Can the ionosphere regulate magnetospheric convection?, J. Geophys. Res., 78, 2837-2851, 1973.

Cowley, S. W. H.: Pitch angle dependence of the charge-exchange lifetime of ring current ions, Planet. Space Sci., 25, 385-393, doi:10.1016/0032-0633(77)90054-X, 1977.

Cowley, S. W. H. and Lockwood, M.: Excitation and decay of solar wind-driven flows in the magnetosphere-ionosphere system, Ann. Geophys., 10, 103-115, 1992, http://www.ann-geophys.net/10/103/1992/.

Crowley, G. and Hackert, C. L.: Quantification of High Latitude Electric Field Variability, Geophys. Res. Lett., 28, 2783-2786, 2001.

Daglis, I. A., Thorne, R. M., Baumjohann, W., and Orsini, S.: The terrestrial ring current: Origin, formation, and decay, Rev. Geophys., 37, 407-438, 1999.

De La Beaujardiere, O., Alcayde, D., Fontanari, J., and Leger, C.: Seasonal dependence of high-latitude electric fields, J. Geophys. Res., 96, 5723-5735, 1991.

Dungey, J. W.: Interplanetary magnetic field and the auroral zones, Phys. Rev. Lett., 6, 47-48, 1961.

Friedrich, E., Rostoker, G., Connors, M. G., and McPherron, R. L.: Influence of the substorm current wedge on the Dst index, J. Geophys. Res., 104, 4567-4576, 1999.

Haaland, S. E., Paschmann, G., Förster, M., Quinn, J. M., Torbert, R. B., McIlwain, C. E., Vaith, H., Puhl-Quinn, P. A., and Kletzing, C. A.: High-latitude plasma convection from Cluster EDI measurements: Method and IMF-dependence, Ann. Geophys., 25, 239-253, 2007, http://www.ann-geophys.net/25/239/2007/.

Hairston, M. R., Drake, K. A., and Skoug, R.: Saturation of the ionospheric polar cap potential during the October-November 2003 superstorms, J. Geophys. Res., 110, A09S26, doi:10.1029/ 2004JA010864, 2005.

Heppner, J. P. and Maynard, N. C.: Empirical high-latitude electric field models, J. Geophys. Res., 92, 4467-4489, 1987.

Hill, T. W.: Mercury and Mars: The role of ionospheric conductivity in the acceleration of magnetospheric particles, Geophys. Res. Lett., 3, 429-432, 1976.

Matsui, H., Quinn, J. M., Torbert, R. B., Jordanova, V. K., PuhlQuinn, P. A., and Paschmann, G.: IMF BY and the seasonal dependences of the electric field in the inner magnetosphere, Ann. Geophys., 23, 2671-2678, 2005, http://www.ann-geophys.net/23/2671/2005/.

Milan, S. E., Baddeley, L. J., Lester, M., and Sato, N.: A seasonal variation in the convection response to IMF orientation, Geophys. Res. Lett., 28, 471-474, 2001.

Nagatsuma, T.: Diurnal, semiannual, and solar cycle variations of solar windmagnetosphereionosphere coupling, J. Geophys. Res., 111, A09202, doi:10.1029/2005JA011122, 2006. 
O'Brien, T. P. and McPherron, R. L.: An empirical phase space analysis of ring current dynamics: Solar wind control of injection and decay, J. Geophys. Res., 105, 7707-7719, doi:10.1029/ 1998JA000437, 2000.

Papitashvili, V. O. and Rich, F. J.: High-latitude ionospheric convection models derived from Defense Meteorological Satellite Program ion drift observations and parameterized by the interplanetary magnetic field strength and direction, J. Geophys. Res., 107, 1198, doi:10.1029/2001JA000264, 2002.

Reiff, P. H. and Burch, J. L.: IMF By-dependent plasma flow and Birkeland currents in the dayside magnetosphere, 2. A global model for northward and southward IMF, J. Geophys. Res., 90, 1595-1609, 1985.

Reiff, P. H. and Heelis, R. A.: Four cells or two? Are four convection cells really necessary?, J. Geophys. Res., 99, 3955-3960, 1994.

Reiff, P. H., Spiro, R. W., and Hill, T. W.: Dependence of polar cap potential drop on interplanetary parameters, J. Geophys. Res., 86, 7639-7648, 1981.

Rich, F. J. and Hairston, M. R.: Large-scale convection patterns observed by DMSP, J. Geophys. Res., 99, 3827-3844, 1994.

Rostoker, G., Savoie, D., and Phan, T. D.: Response of magnetosphere-ionosphere current systems to changes in the interplanetary magnetic field, J. Geophys. Res., 93, 8633-8641, 1988.

Ruohoniemi, J. M. and Greenwald, R. A.: Observations of IMF and seasonal effects in high-latitude convection, Geophys. Res. Lett., 9, 1121-1124, 1995.
Ruohoniemi, J. M. and Greenwald, R. A.: Dependencies of high-latitude plasma convection: Consideration of interplanetary magnetic field, seasonal, and universal time factors in statistical patterns, J. Geophys. Res., 110, A09204, doi:10.1029/ 2004JA010815, 2005.

Russell, C. T. and McPherron, R. L.: The magnetotail and substorms, Space Sci. Rev., 15, 205-266, 1973.

Siscoe, G. L., Formisano, V., and Lazarus, A. J.: Relation between geomagnetic Sudden Impulses and solar wind pressure changes An experimental investigation, J. Geophys. Res., 73, 4869-4874, 1968.

Siscoe, G. L., Crooker, N. U., and Siebert, K. D.: Transpolar potential saturation: Roles of region 1 current system and solar wind ram pressure, J. Geophys. Res., 107, 1321, doi:10.1029/ 2001JA009176, 2002.

Sonnerup, B. U. Ö.: Magnetopause reconnection rate, J. Geophys. Res., 79, 1546-1549, 1974.

Tanaka, T.: Interplanetary magnetic field By and auroral conductance effects on high-latitude ionospheric convection patterns, J. Geophys. Res., 106, 24 505-24 516, 2001.

Weimer, D. R.: Models of high latitude electric potentials derived with a least error fit of spherical harmonic coefficients, J. Geophys. Res., 100, 19595-19607, 1995.

Weimer, D. R.: An improved model of ionospheric electric potentials including substorm perturbations and application to the Geospace Environment Modeling November 24, 1996, event, J. Geophys. Res., 106, 407-416, 2001. 